\title{
A spectroscopic analysis of the most polarizing atomic lines of the second solar spectrum ${ }^{\star}$
}

\author{
L. Belluzzi and E. Landi Degl'Innocenti
}

\author{
Dipartimento di Astronomia e Scienza dello Spazio, University of Firenze, Largo E. Fermi, 2 - 50125 Firenze, Italy \\ e-mail: belluzzi@arcetri.astro.it
}

Received 17 June 2008 / Accepted 18 September 2008

\section{ABSTRACT}

\begin{abstract}
We present an analysis of the second solar spectrum, as plotted in graphical form in the three volumes of the atlas "The Second Solar Spectrum", in the wavelength range between $3160 \AA$ and $6995 \AA$. All the strongest positive linear polarization signals produced by atomic spectral lines are identified, and their amplitudes are listed, along with the most relevant spectroscopic properties of the lines. The various signals are divided into five classes according to the shape of their $Q / I$ profiles. Three empirical rules are formulated from the analysis of the amplitude and shape of the various signals, and of the spectroscopic properties of the spectral lines.
\end{abstract}

Key words. atomic data - line: profiles - polarization - scattering - Sun: atmosphere

\section{Introduction}

The theoretical interpretation of the "second solar spectrum", namely the linearly polarized spectrum of the solar radiation coming from the quiet solar atmosphere close to the limb, is presently one of the most interesting topics in solar physics. The term second solar spectrum, first suggested by Ivanov, is indeed appropriate since this spectrum has a structural richness that can be compared to that of the intensity spectrum, and, at the same time, it shows a significantly different aspect. The first detection of a linear polarization signal in a spectral line observed close to the solar limb (Ca I at $4227 \AA$ ) dates back to 1941 (Redman 1941). After Redman's discovery, other strongly polarizing lines were detected during the 1960s and 1970s (Brückner 1963; Stenflo 1974; Wiehr 1975, 1978). The first systematic analysis was performed by Stenflo and collaborators in the $1980 \mathrm{~s}$ (Stenflo et al. 1980, 1983a,b), though with instruments of reduced polarimetric sensitivity. Most of the spectral structures of the second solar spectrum therefore remained inaccessible until about one decade ago, when the first instruments capable of performing observations with polarimetric sensitivities better than $10^{-3}$ were developed (Stenflo \& Keller 1996, 1997). Today the second solar spectrum is available in graphic form in the atlas “The Second Solar Spectrum" (Gandorfer 2000, 2002, 2005), which covers the spectral range between $3160 \AA$ and $6995 \AA$.

It was soon realized that the second solar spectrum provides a new formidable window for diagnosing the Sun. Its interpretation, which represents an extraordinary theoretical challenge, seems to be a necessary and unavoidable step to improve our knowledge of the solar atmosphere. Although the fundamental physical process at the origin of the second solar spectrum (resonance scattering) is well understood, the formulation of a complete theory, able to describe the generation and transfer of polarized radiation in such a complex environment as the solar atmosphere, has revealed to be an extremely complex

* Tables 1 and 2 are only available in electronic form at http://www . aanda.org task. Today, we can rely on a rigorous theory of the polarization in spectral lines which has been deduced from the principles of quantum electrodynamics (see the monograph by Landi Degl'Innocenti \& Landolfi 2004). Through the application of this theory, combined with the development of suitable numerical methods in polarized radiative transfer (Trujillo Bueno 2003a; Manso Sainz \& Trujillo Bueno 2003), it has been possible to interpret several important features of the second solar spectrum, and to start exploiting it to investigate the physical properties of our nearest star (see Trujillo Bueno 2009, for a recent review).

The interpretation of the second solar spectrum through the above mentioned theory, as well as through other theoretical approaches that have been proposed is, however, still rather fragmentary. The reason is that, as previously observed, the problem we are facing is very complex, and either the approaches based on simplifying assumptions (last scattering approximation, flat spectrum approximation, two-level atom, unpolarized lower level, etc.), or the more sophisticated ones including detailed radiative transfer cannot be applied with success to whatever spectral line. As a consequence, most of the investigations have been dedicated to the interpretation of particular lines or multiplets of this spectrum, while not much attention has been paid to the analysis of its general properties.

In this respect, probably one of the most important questions concerning the whole second solar spectrum, that still waits for an answer, is why only particular lines, of certain elements, produce strong polarization signals. For instance, one can wonder why some elements are particularly present with their lines in the second solar spectrum, whereas other elements of comparable abundance are totally absent. Moreover, observing the second solar spectrum one can easily realize that several conspicuous polarization signals are produced by relatively weak lines in the intensity spectrum, often belonging to atomic or molecular species with low abundances. On the other hand, many strong lines in the intensity spectrum are quite anonymous in the second solar spectrum. At the moment it is difficult to explain in 
a quantitative way such peculiarities, and to predict which lines are expected to produce particularly strong polarization signals, though, when dealing with single spectra, interesting and encouraging, even if qualitative, results have been obtained for Ti I (Manso Sainz \& Landi Degl'Innocenti 2002), and Ce II (Manso Sainz et al. 2006), two of the most remarkable elements of the second solar spectrum.

In this paper we present an investigation carried out over the whole second solar spectrum as plotted in graphical form in the three volumes of the atlas "The Second Solar Spectrum" (Gandorfer 2000, 2002, 2005). The aim is to try to answer the previous question, looking for possible general physical properties common to all the most polarizing atomic spectral lines. The first part of the investigation has been dedicated to a complete analysis of the spectrum, through which we could identify, measure and classify all the strongest polarization signals due to atomic transitions (Sect. 2). We then selected only the atomic spectral lines of unambiguous identification and free of blends, and we analyzed their spectroscopic properties (Sect. 3). This analysis lead us to the formulation of three empirical rules presented in Sect. 4. In Sect. 5 we tried to interpret some of the rules within the framework of the theory presented in Landi Degl'Innocenti \& Landolfi (2004).

\section{Analysis of the second solar spectrum}

We analyzed the second solar spectrum as observed by Gandorfer (2000, 2002, 2005) between 1999 and 2003 using the Zürich Imaging Polarimeter (ZIMPOL) at the Gregory Coudé Telescope of IRSOL (Vols. I and II), and at the McMath-Pierce telescope of Kitt-Peak (Vol. III), in the spectral range between $3160 \AA$ and $6995 \AA$. We decided to refer to these data since the main aim of our investigation was to analyze the full second solar spectrum, and it was therefore essential to refer to a set of homogeneous observations obtained, as much as possible, with the same instrument, and in a relatively short time interval (with respect to the solar activity cycle). However, it is important to point out that several signals that are not so conspicuous in Gandorfer's atlas, turned out to be much stronger in other observing runs, made in different periods of the activity cycle ${ }^{1}$.

Though the second solar spectrum contains many polarization signals due to molecular transitions, in this investigation we limited ourselves to the analysis of the atomic lines. We then identified all the atomic spectral lines producing positive linear polarization signals (according to the specifications better defined in Sect. 2.2) with an amplitude larger than $0.1 \%$. To take into account the decrease of the polarization degree, both in the continuum and in the spectral lines, going towards the red part of the visible spectrum, this threshold value has been decreased to $0.05 \%$ for wavelengths larger than $4500 \AA$. These threshold values for the $Q / I$ signal amplitude, as well as the wavelength limit of $4500 \AA$, are completely arbitrary. Although more sophisticated criteria could in principle be applied, our choice seems in any case to be quite reasonable: in the analyzed spectral range, in fact, about 250 lines producing polarization signals higher than the threshold values can be identified. This number is statistically significant and, at the same time, still easily manageable.

\footnotetext{
${ }^{1}$ A particularly relevant example is the one of the Mg I b-lines. The polarization signals observed in these lines by Stenflo and collaborators in 1995 (see Stenflo et al. 2000) are significantly different from those of Gandorfer's atlas.
}

\subsection{Classification of the $Q / /$ signals}

We divided all the selected signals into three main classes, according to the shape of the $Q / I$ profile. The signals which show a $Q / I$ profile with a single peak at line center have been classified as "S" signals (see Fig. 1, panel a), those which depolarize in the wings, and produce a narrow peak at line center have been classified as "W" signals (see Fig. 1, panel b), while those that polarize in the wings, show a decrease of the polarization amplitude approaching to the line-core and, eventually, show a narrow peak at line center have been classified as "M" signals. The "M" signals have been further divided into three subclasses, according to the presence or not of the line-core peak, and according to the relative width of the wing-lobes with respect to the line-core peak (if present). The "M" signals which do not show any polarizing peak at the line-core (or that show a weak line-core peak, with an amplitude below the threshold value) have been classified as "M0" (see Fig. 1, panels c and d), those which show in the wings polarization lobes much broader than the line-core peak have been classified as "M1" (see Fig. 1, panels e and f), while those which show in the wings polarization peaks whose width is similar to, or smaller than, the width of the line-core peak have been classified as "MS" (see Fig. 1, panels g and h). As far as "M1" and "MS" signals are concerned, our classification scheme does not take into account whether the line-core peak exceeds or not the continuum level or the wing-lobe amplitude. We have to remark that the classification of the signals through these criteria can be quite ambiguous in some cases. However, we believe that the scheme presented in this paper can be considered as a good starting point to organize in a few significative classes the wide variety of polarimetric signals that are found in the second solar spectrum. Examples of signals of the second solar spectrum belonging to the various classes proposed can be found in Fig. 2.

Some "S" signals lie on the large wings of strongly depolarizing lines. In these cases, if the peak does not reach the theoretical continuum, it is not trivial to understand if we are in the presence of a polarizing spectral line or not. Indeed, if the line is intrinsically "neutral", or less depolarizing than the wings of the strong line, the resulting peak would be nothing but a kind of an "artefact" (see Fig. 3, panel a). In this respect, important hints can be obtained from the analysis of other possible blends present in the same depolarizing wings. For example, if all the blended lines produce polarization peaks, and these are very similar to each other, very likely their polarization effect is only apparent, and they have not been taken into account. On the other hand, if we are in the presence of such a blend, and there are reasons to believe that the polarization signal is "real", then it has been classified as " $\mathrm{S}$ ".

At wavelengths shorter than $4000 \AA$, the solar spectrum is crowded by a large number of very intense spectral lines, usually strongly blended between each other. A significant number of these strong lines produce in the second solar spectrum strong polarization signals, which very often overlap. This peculiarity makes our investigation rather difficult in this spectral region. On one hand, in fact, it is not unusual to find interesting polarization signals strongly blended with the large wings of these lines, and in these cases it can be quite difficult to classify the shape of the signals, and to decide whether or not they exceed the threshold value. In several cases we tried to justify our conclusions with a note in Table 1 (see Sect. 2.3). On the other hand, the classification of the polarization signals produced by the same strong lines is in some cases rather difficult. If they are isolated, in the sense that their extended wings are not blended with other 


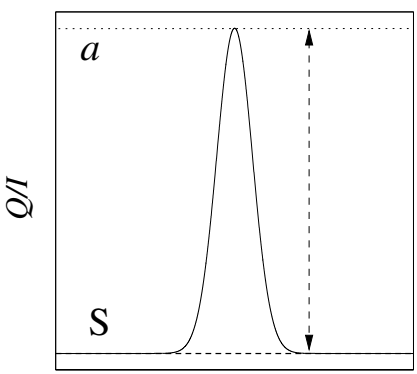

$\lambda$

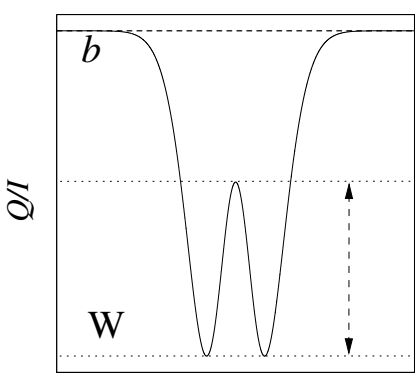

$\lambda$

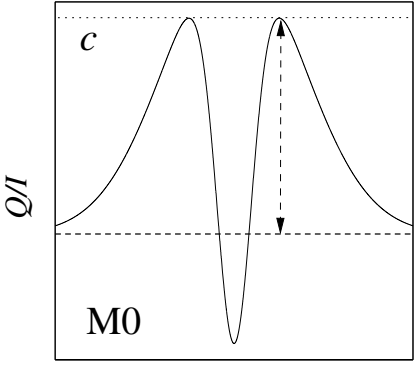

$\lambda$

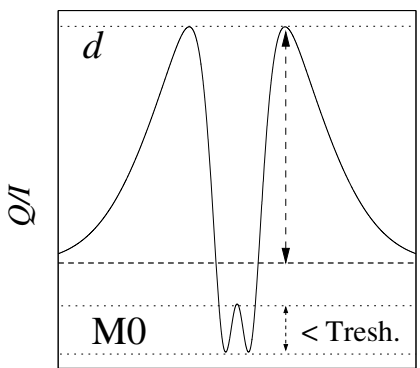

$\lambda$

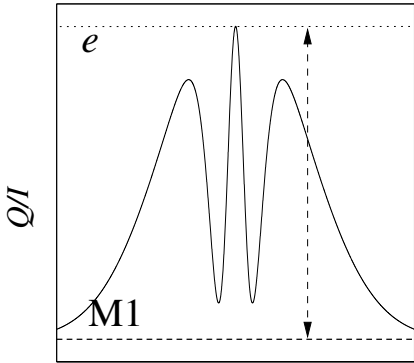

$\lambda$

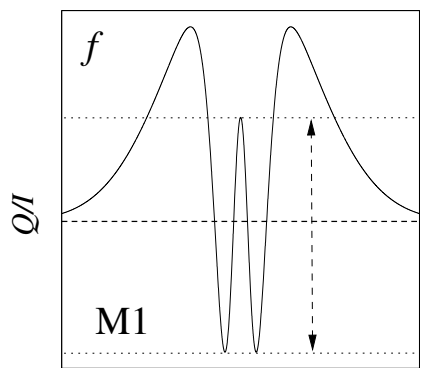

$\lambda$

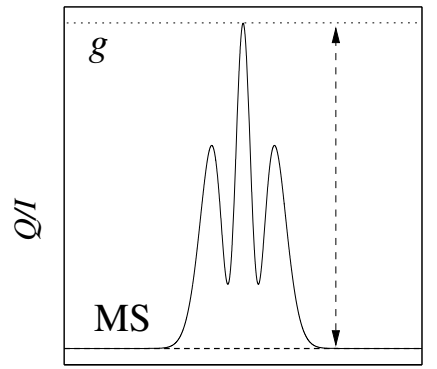

$\lambda$

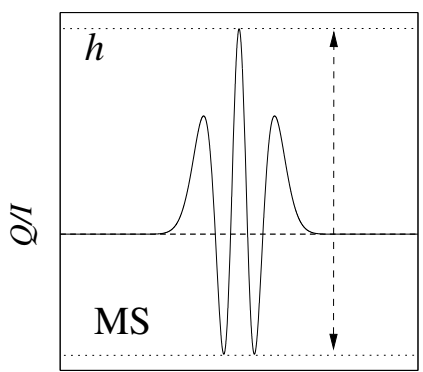

$\lambda$

Fig. 1. Classification scheme of the $Q / I$ profiles. The thick dashed horizontal line represents the continuum level. The vertical dashed line joins the limits between which the amplitude of the signal has been measured.
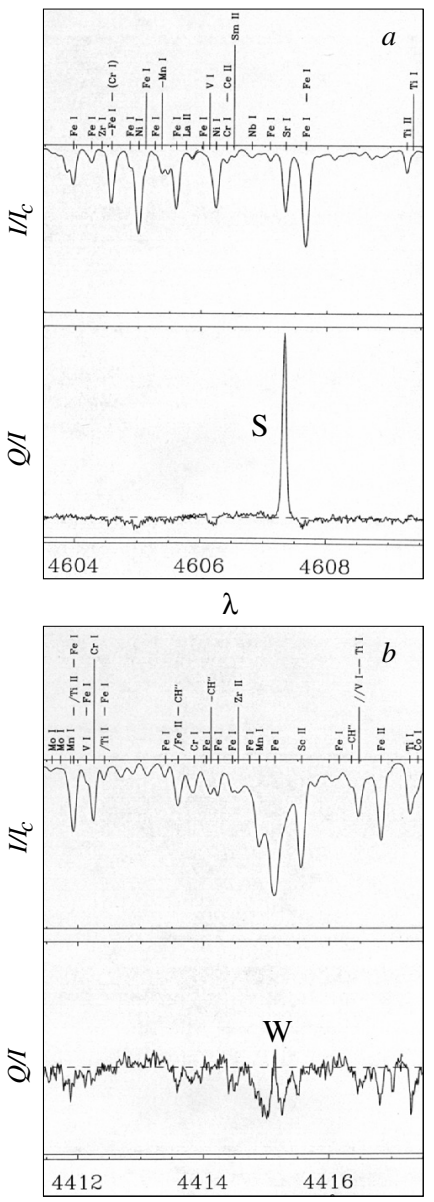

$\lambda$
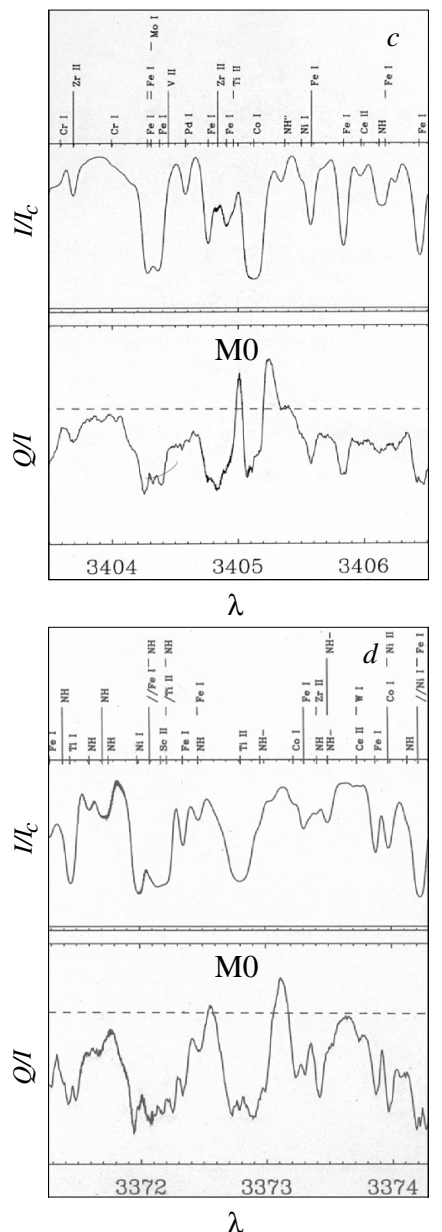
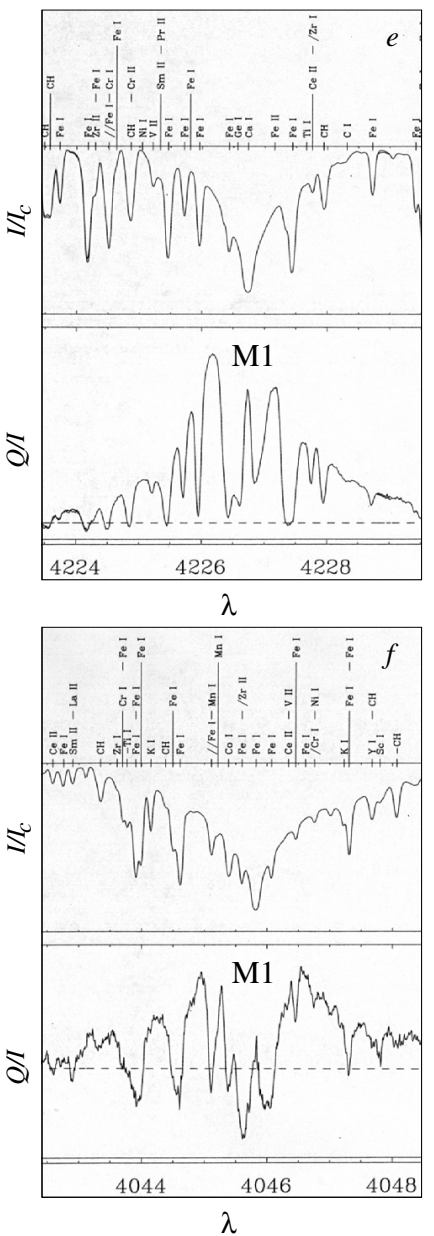
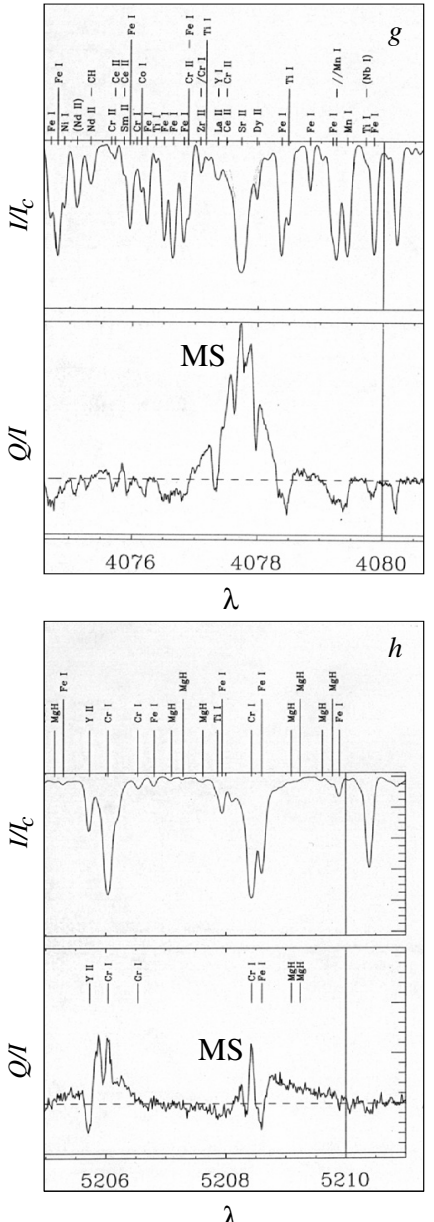

Fig. 2. Examples of signals of the second solar spectrum belonging to the various classes proposed. Panel a): Sr I at $4607 \AA$ (S signal); panel b): Fe I at $4415 \AA$ (W signal); panels c) and d): Co I at $3405 \AA$ and Ti II at $3373 \AA$ (M0 signals), panels e) and f): Ca I at $4227 \AA$ and Fe I at $4046 \AA$ (M1 signals); panels g) and h): Sr II at $4078 \AA$ and Cr I at $5208 \AA$ (MS signals). The observations shown in this figure have been scanned from the atlas "The Second Solar Spectrum" (Gandorfer 2000, 2002, 2005). 


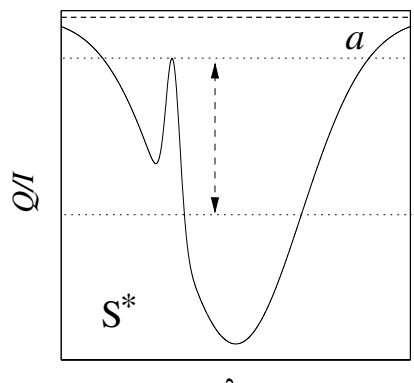

$\lambda$

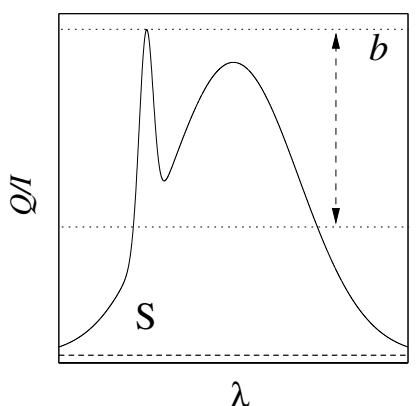

$\lambda$

Fig. 3. Examples of polarization signals blended with stronger and broader ones. Panel a): a $S^{*}$ signal. Panel b): amplitude measurement of a $\mathrm{S}$ signal blended with the blue wing of a stronger one.

strong lines belonging to the same multiplet, then the signals can be usually classified following the proposed scheme (usually as "M0" or "M1"). On the contrary, if blends between strong lines of the same multiplet are present, then in the far wings of the lines the polarization is mainly due to quantum interferences between different $J$-levels (see for example the $\mathrm{H}$ and $\mathrm{K}$ lines of $\mathrm{Ca}$ II), and the resulting profiles might not fall in one of the classes proposed.

\subsection{Measurement of the signal amplitude}

With the exception of the "M0" lines, what has been measured and compared to the threshold value is the amplitude of the linecore peak. We believe, in fact, that this quantity contains the most interesting physical information. Moreover, it is rather uncommon for this peak to be severely affected by blends, since it falls at line center. As far as the "S" signals are concerned, the amplitude of the peak is measured from the continuum level which is plotted in the atlas (see Fig. 1, panel a). We recall that this quantity is not directly observed, but it is deduced from the theoretical calculations of Fluri \& Stenflo (1999), for $\lambda>4000 \AA$, and of Stenflo (2005) for $\lambda<4000 \AA$. On the other hand, as far as "W" signals are concerned, the amplitude of the peak is measured from the lowest depolarizing dip (see Fig. 1, panel b). Concerning the "M1" and "MS" signals, the choice of the reference level from which the line-core peak amplitude is measured depends on whether the polarization at the lowest depolarizing dip is below the theoretical continuum or not. In the former case it is measured from the lowest depolarizing dip (see Fig. 1, panels $\mathrm{f}$ and $\mathrm{h}$ ), in the latter from the theoretical continuum (see Fig. 1, panels e and g). As far as the "M0" signals are concerned, what has been measured, and compared to the threshold value, is the amplitude of the wing-lobes with respect to the theoretical continuum (see Fig. 1, panels $\mathrm{c}$ and d). It is important to note that this quantity has to be considered with caution. The wing-lobes, in fact, are usually very broad, and often the presence of blends makes impossible the estimate of their real amplitude. For the sake of completeness, also for the "M1" and "MS" signals the amplitude of the wing-lobes has been measured and listed in Table 1 (see Sect. 2.3) below the amplitude of the line-core peak.

In the case of blends, the unblended (or less blended) left or right dip (or lobe) has been considered. The amplitude of any "S" signal blended with a stronger (polarizing or depolarizing) signal is not measured from the theoretical continuum of the atlas, but from a reference value qualitatively estimated from the expected shape of the $Q / I$ profile of the blending line (see Fig. 3 , panels a and $b$ ).

\subsection{Table of the most polarizing atomic spectral lines of the second solar spectrum}

In Table 1 we list, in order of increasing wavelength, all the atomic spectral lines that produce positive polarization signals higher than the threshold value $(0.1 \%$ if $\lambda<4500 \AA$, or $0.05 \%$ if $\lambda \geq 4500 \AA$ ), as explained in detail in Sect. 2. In the first four columns we list the wavelength (in $\AA$ ), the element (elements in the case of unresolved blends), the equivalent width (in $\mathrm{m} \AA$ ), and the reduced equivalent width (in Fraunhofer). All these quantities have been taken from Moore et al. (1966). In the case of blends the same notation as in this reference monograph has been used: if one component of a blend dominates with respect to the other components, its chemical symbol is preceded by " $/ /$ ", if it contributes more than the others, it is preceded by "/. If one component is completely hidden by the others, its chemical symbol is written in brackets. If two or more components are separated by a dash, this means that the first one falls at a shorter wavelength than the second one. If there is only one component preceded or followed by a dash, this indicates that the line is probably a blend, the other unknown contributor being, respectively, at a shorter or longer wavelength. A "p" following the chemical symbol indicates that the spectral line is "predicted" from known atomic energy levels, but it has not been observed in the laboratory yet. An asterisk over the numerical value of the equivalent width means that it is referred to the blend of two or more lines, one of which is the one under investigation. The reduced equivalent width is known for all the selected lines but one (Ba I at $5535.51 \AA$ ), and it is often derived through theoretical procedures (see Moore et al. 1966, for details).

In the next 3 columns we list the value of the amplitude of the polarization signal (in percent), giving the values of $(Q / I)^{M}$, $(Q / I)^{m}$, and the difference $Q / I=(Q / I)^{M}-(Q / I)^{m}$. The quantities $(Q / I)^{M}$ and $(Q / I)^{m}$ are the values of $(Q / I)$ measured at the top and at the bottom of the polarization signal, respectively. As far as "S" signals are concerned, an asterisk over the value of $(Q / I)^{m}$ indicates that this reference polarization value is not the theoretical continuum plotted in the atlas, but a value estimated by the authors as previously described, because of the presence of a blend. In the case of "M1" and "MS" signals we list both the amplitude of the line-core peak (which is the quantity we will refer to throughout our investigation) and below, for the sake of completeness, the amplitude of the wing-lobes. This last quantity has not been listed in the case of severely blended wing-lobes. Note that the amplitude of the wing-lobes, both for the "M0" signals, and for the "M1" and "MS" signals, has been quoted in italic. These three quantities, as explained, have been measured graphically on the three volumes of the atlas "The Second Solar Spectrum" (Gandorfer 2000, 2002, 2005). Finally, in the last column we quote the classification of the $Q / I$ profile, according the scheme described in Sect. 2.1. Several notes have been added with the aim of clarifying either our classification, or the $Q / I$ measurement, in the case of blended signals, and in order to point out peculiarities that have been noticed in several profiles during our analysis.

\section{Selection of the Lines}

Once the polarization signals exceeding the threshold value have been identified, classified, and listed in Table 1, we concentrated only on those produced by spectral lines free of blends in the intensity spectrum, and with unambiguous classification and 
amplitude measurement (signals free of blends in the $Q / I$ spectrum). These lines are collected, element by element, in Table 2.

In the first six columns of Table 2 we list the element, the solar and laboratory wavelengths, the reduced equivalent width, the $Q / I$ signal amplitude, and the classification of the $Q / I$ signal. With the exception of the laboratory wavelengths, which are taken from Moore $(1945)^{2}$, all the other quantities are the same as in Table 1. As far as "S" signals are concerned, an asterisk over the $Q / I$ value indicates that the amplitude of the peak has not been measured from the theoretical continuum, but from another reference value because of the presence of a blend, as explained in detail in Sect. 2.2.

In the next two columns we list the lower and upper terms of the transition, the multiplet number, and the $J$-values of the lower and upper levels of the transition. These quantities are taken from Moore $(1945)^{2}$. In column nine we list the Einstein coefficients for spontaneous emission. These quantities are taken either from Ralchenko et al. (2008) or from Kurucz \& Bell (1995), depending on which database quotes the more recent work.

In the next four columns we list the energies and the Landé factors of the lower and upper levels. These values, as well as the percentage of the coupling scheme listed in the last column, are taken from Moore $(1949,1958,1971)$, and from Martin et al. (1978). The only exception are the hydrogen lines. In these cases the energies, which are the average values over the fine structure, are taken from Kurucz \& Bell (1995). An asterisk over the Landé factor indicates that the value is not the experimental one, but is calculated theoretically under the hypothesis of $L-S$ coupling. As far as hydrogen is concerned, it has been demonstrated that the Landé factor of all its levels is equal to 1 (Casini \& Landi Degl'Innocenti 1994). In the following column we specify if the lower level is the ground level (GL), a metastable level (ML), or a level that, at the same time, is the upper level of a transition that produces a polarization signal entering our threshold values (PL). A question mark (PL?) means that the lower level is the upper level of a transition producing a spectral line that falls outside the wavelength interval covered by the atlas, but for which there are hints to believe that it might produce a strong signal in the second solar spectrum. A short dash in this column means that the lower level does not enter any of these three classes (there is just one line of this kind). Hereafter, following the conventions of spectroscopy, the transition whose lower level is the upper level of another transition, will be referred to as a "subordinate transition", while the transition whose lower level is the ground level, or a metastable level, will be referred to as a "resonance transition". A level is considered metastable if it cannot be connected through an allowed transition (according to the selection rules in the $L-S$ coupling scheme) to a lower level, in other words, if there are no lower levels showing opposite parity, and satisfying the selection rules $\Delta \ell= \pm 1, \Delta S=0$, $\Delta L=0, \pm 1,0 \rightarrow 0, \Delta J=0, \pm 1,0 \rightarrow 0$. The lower level of five Ce II lines (the first four lines, and the last line of this ion listed in Table 2) is not metastable according to the previous definition. For these levels, however, $L-S$ coupling holds only approximately, and the allowed transitions towards lower levels fall in the infrared part of the spectrum, at wavelengths larger than $20000 \AA$ ( $11500 \AA$ as far as the level at $11015.579 \mathrm{~cm}^{-1}$ is concerned). Unfortunately there are no data concerning the transition probabilities and the relative intensities of these infrared lines, but we believe that it should be rather correct to consider

2 The data for the $\mathrm{Nb}$ II and the Dy II lines are taken from Kurucz \& Bell (1995).
Table 3. Lines of Table 2 produced by subordinate transitions, and corresponding resonance lines.

\begin{tabular}{cccc|ccc}
\hline \hline & \multicolumn{3}{c|}{ Subordinate trans. } & \multicolumn{3}{|c}{ Resonant trans. } \\
El. & $\lambda$ & $Q / I$ & type & $\lambda$ & $Q / I$ & type \\
& $(\AA)$ & $(\%)$ & & $(\AA)$ & $(\%)$ & \\
\hline $\mathrm{H} \mathrm{I}$ & 4340.475 & 0.12 & $\mathrm{~W}$ & $1216\left[\mathrm{Ly}_{\alpha}\right]$ & outside \\
& 6562.808 & 0.109 & $\mathrm{~S}$ & $1216\left[\mathrm{Ly}_{\alpha}\right]$ & atlas range \\
$\mathrm{Mg}$ I & 5528.418 & 0.052 & $\mathrm{~W}$ & 2852 & outside \\
& 4703.003 & 0.050 & $\mathrm{~W}$ & 2852 & atlas range \\
$\mathrm{Ca}$ II & 3179.342 & 0.35 & $\mathrm{~W}$ & 3933.682 & 0.90 & $\mathrm{M} 1$ \\
$\mathrm{Fe}$ I & 6024.068 & 0.05 & $\mathrm{~S}$ & 4045.825 & 0.390 & $\mathrm{M} 1$ \\
$\mathrm{Cu}$ I & 5218.209 & 0.052 & $\mathrm{~S}$ & 3247.540 & 0.93 & $\mathrm{M} 0$ \\
$\mathrm{Ba}$ II & 4130.657 & 0.28 & $\mathrm{~S}$ & 4554.036 & 0.65 & $\mathrm{MS}$ \\
\hline
\end{tabular}

these levels metastable as well. Finally, in the last column we list, when available, the percentage of the coupling schemes of the upper and lower terms given in column seven. We recall that light elements are usually well described by the $L-S$ coupling scheme. For the sake of completeness, if a signal, that has not been selected (because of blends in the intensity spectrum or in the second solar spectrum), is produced by a transition that belongs to a multiplet already present in Table 2, this transition has been listed in italic fonts in the same table.

\section{Results}

In the spectral range between $3190 \AA$ and $6995 \AA$, we could identify 248 polarimetric signals, due to atomic transitions, that exceed the threshold value. The signals that can be unambiguously associated to a particular transition (no blends in the intensity spectrum), and that can be unambiguously classified and measured (no blends in the second solar spectrum) are 158. Hereafter we will always refer only to these lines (listed in roman fonts in Table 2).

Our analysis allows establishing some empirical laws. The first one can be stated as follows. Rule 1: The lower level of the transitions corresponding to the 158 lines listed in Table 2, namely the most prominent lines of the second solar spectrum, is either the ground level or a metastable level (in the sense specified above) or, alternatively, the upper level of a resonance transition corresponding to a line also contained in the same table. In other words, all the lines of Table 2 are either resonance lines, or subordinate lines sharing their lower level with the upper level of a resonance line producing a strong polarization signal. This rule has only one exception, the Ba II line at $3891.781 \AA$. The lower level of the corresponding transition $\left(6 \mathrm{p}^{2} \mathrm{P}_{1 / 2}^{\mathrm{o}}\right)$, in fact, is the upper level of the transition corresponding to the Ba II $\mathrm{D}_{1}$ line at $4934.095 \AA$, which shows a depolarization feature in the atlas of Gandorfer (2000), and of the transition $5 \mathrm{~d}^{2} \mathrm{D}_{3 / 2}-6 \mathrm{p}^{2} \mathrm{P}_{1 / 2}^{\mathrm{o}}$ at $6496.908 \AA$, which is a weakly polarizing line (see Gandorfer 2000).

Without considering the above mentioned Ba II line, only eight lines of Table 2 are produced by subordinate transitions. As stated in Rule 1, the lower level of these transitions is always the upper level of a resonance one. Subordinate lines, and their corresponding resonance lines are listed in Table 3. In four cases the corresponding resonance transition produces a polarization signal included in Table 2, in four cases it falls outside the spectral interval covered by the three volumes of the atlas. These are the $\operatorname{Ly} \alpha \mathrm{H}$ I line at $1216 \AA$, and the $\mathrm{Mg}$ I resonance line at $2852 \AA\left({ }^{1} \mathrm{~S}_{0}-{ }^{1} \mathrm{P}_{1}\right)$. Although the second solar spectrum of these two lines has not been observed yet, it is expected that they 

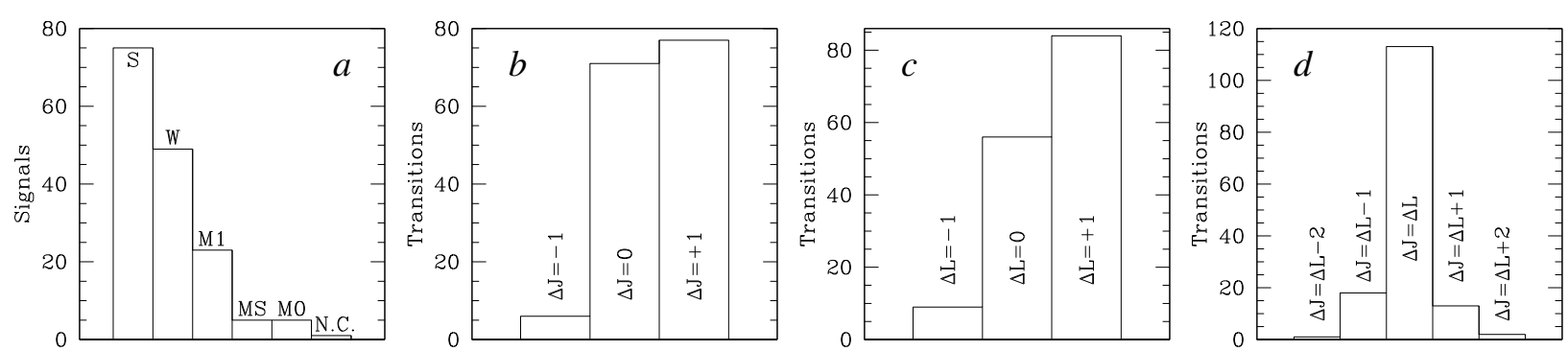

Fig. 4. Histograms showing: a) the number of signals in each class; b) the number of transitions with $\Delta J= \pm 1,0$; $\mathbf{c}$ ) the number of transitions with $\Delta L= \pm 1,0$; and d) the number of transitions with $\Delta J-\Delta L= \pm 2, \pm 1,0$. The unresolved fine structure transitions are not included in the histogram of panel b) (H I lines, and Mg I lines at $3832.310 \AA$, and $3838.302 \AA$ ). The transitions between terms that are not described within the $L-S$ coupling scheme are not included in the histogram of panel c). The transitions excluded from the histograms of panels $\mathbf{b})$ and $\mathbf{c}$ ) are also excluded from the histogram of panel d).

might produce strong polarization signals. In particular, as far as the $\mathrm{Mg}$ I line is concerned, this conclusion comes from the fact that $\mathrm{Ca} \mathrm{I}, \mathrm{Sr}$ I, and $\mathrm{Ba}$ I (elements belonging to the $\mathrm{Mg}$ I isoelectronic sequence) all show a transition ${ }^{1} \mathrm{~S}_{0}-{ }^{1} \mathrm{P}_{1}$, which produces a strong polarization signal in the second solar spectrum (see Table 2). Note in particular that the Ca I line (4226.740 $\AA$ ), and the Sr I line (4607.338 $\AA$ ) produce two of the strongest polarization signals of the second solar spectrum. A detailed analysis of the second solar spectrum of the alkali metals and of the alkali earths metals (and of the ions belonging to their isoelectronic sequences) will be the subject of a future investigation. In the following subsections we discuss some general results concerning the classification of the polarization signals, and the quantum numbers of the corresponding transitions. The results are presented in the form of histograms and scatter plots in Figs. 4 and 5.

\section{1. $Q / /$ profile classification}

From Fig. 4 (panel a), it can be observed that most of the signals are of type "S" and of type "W". The only signal that does not fit into our classification scheme is the one produced by the $\mathrm{Na} \mathrm{I} \mathrm{D}_{1}$ line at $5895.940 \AA$. Plotting the signal amplitude as a function of the reduced equivalent width, and distinguishing through different colors the various classes (see Fig. 5, panel a), a second empirical rule can be established. Rule 2: all the signals produced by the spectral lines of Table 2 having a small equivalent width (i.e. lines having $W_{\lambda} / \lambda<20 F$ ) are of type " $S$ ". The only line, with a large reduced equivalent width, that produces in the second solar spectrum an " $\mathrm{S}$ " signal is the hydrogen line at $6563 \AA\left(\mathrm{H}_{\alpha}\right)$. This line, however, has to be considered with caution within our sample of lines. Its $Q / I$ " $\mathrm{S}$ " profile, in fact, is much broader than all the other " $S$ " signals, and also its intensity profile is quite peculiar. The fact that lines having high values of the equivalent width show profiles of the type "W" or "M", suggests that for these lines transfer effects and partial redistribution effects play an important role.

\subsection{Quantum numbers}

Among the 158 selected lines, a reduced number (18) correspond to transitions which do not satisfy the selection rule $\Delta S=$ $S_{\mathrm{u}}-S_{\ell}=0$ (the indices u and $\ell$ meaning "upper" and "lower"), while 2 correspond to transitions which do not satisfy the selection rule $\Delta L=L_{\mathrm{u}}-L_{\ell}=0, \pm 1,0 \nrightarrow 0$. These lines are listed in Table 4 . Note that as far as rare earth elements are concerned, the
Table 4. Transitions breaking the $\Delta S=0$ and the $\Delta L=0, \pm 1,0 \nrightarrow$ 0 rules.

\begin{tabular}{ccccc}
\hline \hline Elem. & $\begin{array}{c}\lambda \\
(\AA)\end{array}$ & $\Delta S$ & $\begin{array}{c}A \\
\left(10^{7} \mathrm{~s}^{-1}\right)\end{array}$ & Type \\
\hline Ti I & 5565 & -1 & 1.41 & $\mathrm{~S}$ \\
& 4778 & -1 & 1.96 & $\mathrm{~S}$ \\
V II & 3592 & 1 & 4.41 & $\mathrm{~W}$ \\
Fe I & 3521 & -1 & 0.28 & $\mathrm{~W}$ \\
& 3570 & -1 & 6.76 & $\mathrm{M} 1$ \\
& 4384 & 1 & 5.00 & $\mathrm{M} 1$ \\
& 4405 & 1 & 2.75 & $\mathrm{M} 1$ \\
& 4415 & 1 & 1.18 & $\mathrm{~W}$ \\
& 6024 & 1 & 1.27 & $\mathrm{~S}$ \\
Ni I & 3625 & 1 & 0.026 & $\mathrm{~W}$ \\
& 3602 & 1 & 0.044 & $\mathrm{~W}$ \\
& 3453 & 1 & 0.98 & $\mathrm{~W}$ \\
& 3462 & 1 & 2.70 & $\mathrm{~W}$ \\
& 3674 & 1 & 0.20 & $\mathrm{~W}$ \\
& 3485 & -1 & 3.38 & $\mathrm{~S}$ \\
Ce II & 4223 & -1 & 1.87 & $\mathrm{~S}$ \\
Nd II & 4463 & 1 & 2.46 & $\mathrm{~S}$ \\
Dy II & 3695 & 1 & 2.59 & $\mathrm{~S}$ \\
\hline Elem. & $\lambda$ & $\Delta L$ & $\mathrm{~A}$ & Type \\
& $(\AA)$ & & $\left(10^{7} \mathrm{~s}^{-1}\right)$ & \\
\hline Ni I & 3664 & -2 & 0.20 & $\mathrm{~W}$ \\
& 3485 & 2 & 3.38 & $\mathrm{~S}$ \\
\hline
\end{tabular}

terms involved in the transitions cannot be described following exactly the $L-S$ coupling scheme (see Table 2).

As it can be observed from panel b of Fig. 4, most of the signals are produced by transitions with $\Delta J=J_{\mathrm{u}}-J_{\ell}=0$ or $\Delta J=+1$. The six most polarizing lines, in particular, have $\Delta J=+1$, while the lines with $\Delta J=-1$ (six lines, all falling at wavelengths shorter than $4500 \AA$ ) produce in general weak polarization signals, close to the threshold value (see Fig. 5, panel b; note that two points, corresponding to the Ni I lines at $3492.9 \AA$, and $3380.6 \AA$, perfectly overlap so that only five red points can be observed). With the only exception of the Mn I line at $4034.5 \AA$, whose polarization signal reaches an amplitude of $0.17 \%$, all the other lines with $\Delta J=-1$ produce signals that do not exceed $0.11 \%$. A third empirical rule can then be stated as follows. Rule 3: the lines of Table 2 showing the strongest polarization signals $(Q / I>0.17 \%)$ are due to transitions having either $\Delta J=+1$ or $\Delta J=0, \Delta J$ being defined as $J_{\mathrm{u}}-J_{\ell}$. Note finally that all the transitions with $\Delta J=-1$ produce "W" signals.

From panel $\mathrm{c}$ of Fig. 4, it can be observed that most of the signals correspond to transitions having either $\Delta L=+1$ or $\Delta L=0$. The few transitions with $\Delta L=-1$ produce in general 

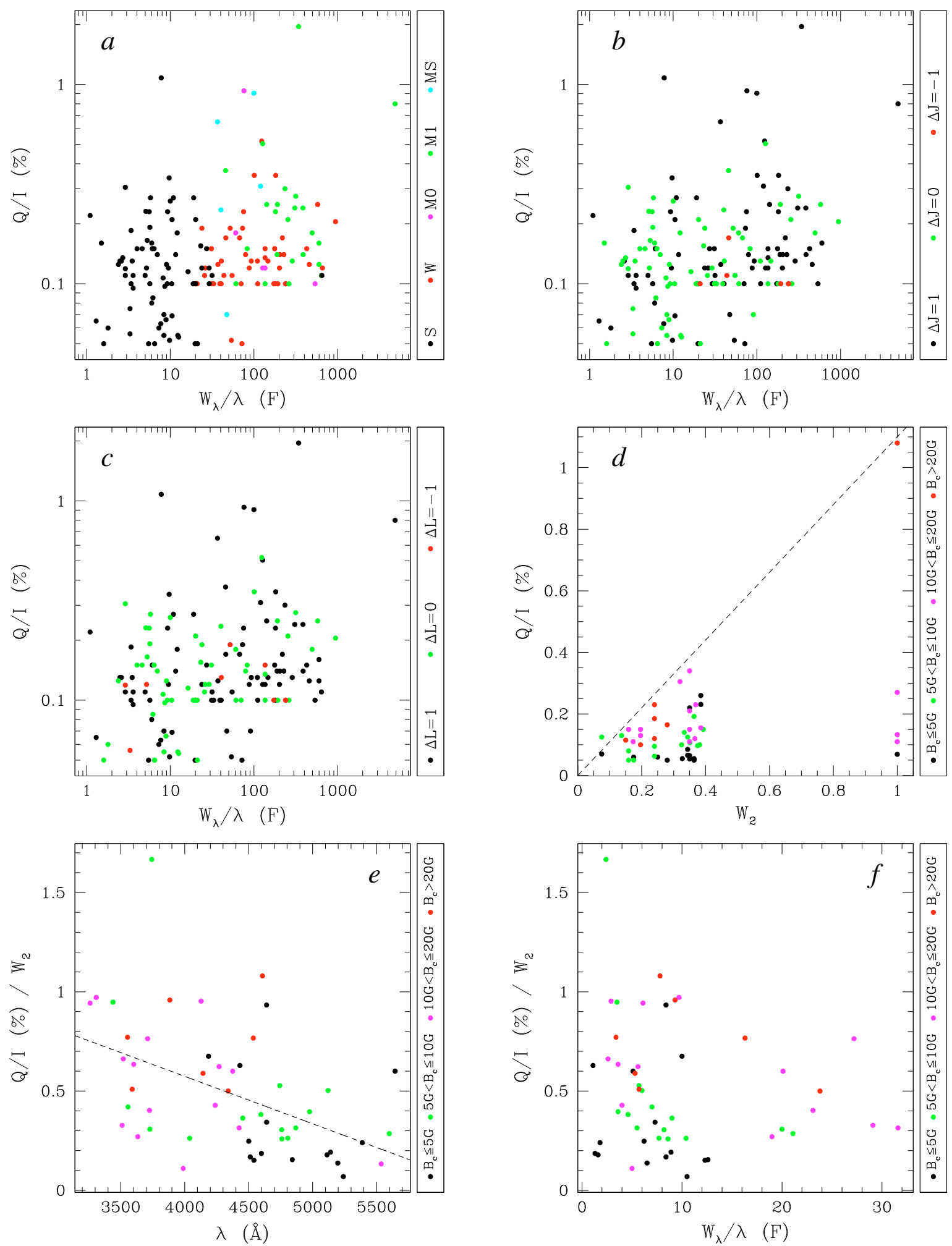

Fig. 5. In the first three graphs (from left to right and from top to bottom) the amplitude of the selected signals is plotted as a function of the reduced equivalent width. The different colors allow distinguishing the signals belonging to the various classes (panel a)), corresponding to transitions with different $\Delta J$ (panel b)), and to transitions with different $\Delta L$ (panel $\mathbf{c})$ ). The lines excluded from the histograms of panels b) and $\mathbf{c})$ of Fig. 4 have been excluded as well from the plots of panels b) and c). In panel d) the signal amplitude is plotted as a function of the quantity $W_{2}($ see Eq. (2) for its definition). The black dashed line has a slope of 0.011: all the points lie either close to this line, or below it. In panel e) the ratio $(Q / I) / W_{2}$ is plotted as a function of wavelength. The black dashed line represents the best linear fit to the points, and shows a decreasing trend with wavelength. In panel f) the same quantity is plotted as a function of the reduced equivalent width. In the last three graphs only the "S" signals (with exception of the $\mathrm{H}_{\alpha}$ line), with metastable (or fundamental) lower level, and with the upper and lower levels described by the $L-S$ coupling scheme (see footnote 3 ) have been considered. The intensity of the critical magnetic field (see text) is represented through a color scale. 
rather weak polarization signals (see Fig. 5, panel c). In the next section we will try to justify this empirical result within the theoretical framework presented in Landi Degl'Innocenti \& Landolfi (2004).

Finally, panel d of Fig. 4 shows that most of the signals are produced by transitions with $\Delta L=\Delta J$. This seems to be due to the fact that within the same multiplet the strongest lines in the intensity spectrum (which, as can be demonstrated, are those with $\Delta L=\Delta J$ ) are those which produce the strongest linear polarization signals in the second solar spectrum.

\section{Theoretical considerations}

In this section we try to interpret some aspects of the results presented above within the framework of the theory presented in Landi Degl'Innocenti \& Landolfi (2004). Since most of the selected lines belong to multiplets, the two-level atom approximation cannot be applied. We consider therefore a two-term atom with unpolarized lower term. If we neglect collisions, and we assume that no magnetic field is present, it can be demonstrated (see Sect. 10.17 of Landi Degl'Innocenti \& Landolfi 2004) that in the neighborhood of a particular line the explicit expression of the emission coefficient in the four Stokes parameters is

$$
\begin{aligned}
\varepsilon_{i}= & k_{M}^{A} S^{J_{\ell}, J_{\mathrm{u}}} \phi\left(v_{\beta_{\mathrm{u}} L_{\mathrm{u}} S J_{\mathrm{u}}, \beta_{\ell} L_{\ell} S J_{\ell}}-v\right) \sum_{K Q} W_{K}\left(L_{\ell} L_{\mathrm{u}} S, J_{\ell} J_{\mathrm{u}}\right) \\
& \times(-1)^{Q} \mathcal{T}_{Q}^{K}(i, \boldsymbol{\Omega}) J_{Q}^{K}\left(v_{\beta_{\mathrm{u}} L_{\mathrm{u}} S, \beta_{\ell} L_{\ell} S}\right) \text { with }(i=I, Q, U, V),
\end{aligned}
$$

where the product $k_{M}^{A} S^{J_{\ell}, J_{\mathrm{u}}}$ is the frequency integrated absorption coefficient relative to a single line of the multiplet, $\phi$ is the line profile, $\mathcal{T}_{Q}^{K}$ is a geometrical tensor, $J_{Q}^{K}$ is a tensor which describes the radiation field illuminating the atomic system, and finally

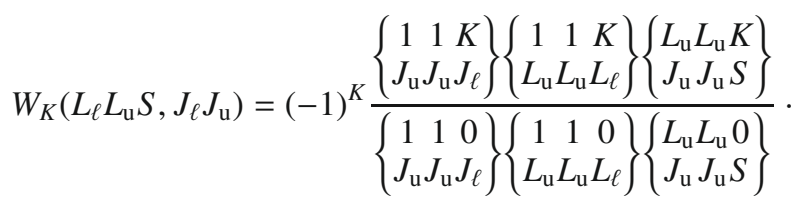

Note that Eq. (1) is obtained assuming that the radiation field tensor, $J_{Q}^{K}$, has the same value for all the transitions of the multiplet. We consider a plane-parallel model of the solar atmosphere, and we take a Cartesian reference system with the $z$ axis (the quantization axis) directed along the vertical. Under this approximation the radiation field illuminating the atomic system has cylindrical symmetry about the vertical, and can be fully described through only two components of the radiation field tensor: $J_{0}^{0}$, and $J_{0}^{2}$. The former describes the average intensity of the radiation field over all the directions of propagation, the latter gives a measure of its anisotropy. The anisotropy degree of the radiation field is usually quantified through the so-called anisotropy factor, a dimensionless parameter defined as $w=\sqrt{2}\left(J_{0}^{2} / J_{0}^{0}\right)$.

Taking the reference direction for positive $Q$ parallel to the atmosphere, the only non zero radiative transfer coefficients are $\eta_{I}, \eta_{Q}, \varepsilon_{I}$ and $\varepsilon_{Q}$, where $\eta_{I}$ and $\eta_{Q}$ are the absorption coefficients in the Stokes parameters $I$ and $Q$, respectively (see Landi Degl'Innocenti \& Landolfi 2004, Eqs. (7.47a-e)). The radiative transfer equations take therefore the simpler form

$\frac{\mathrm{d}}{\mathrm{d} s}\left(\begin{array}{c}I \\ Q\end{array}\right)=-\left(\begin{array}{cc}\eta_{I} & \eta_{Q} \\ \eta_{Q} & \eta_{I}\end{array}\right)\left(\begin{array}{c}I \\ Q\end{array}\right)+\left(\begin{array}{c}\varepsilon_{I} \\ \varepsilon_{Q}\end{array}\right)$

Since the anisotropy degree is small in the solar atmosphere, we have $\eta_{Q} \ll \eta_{I}$ and $\varepsilon_{Q} \ll \varepsilon_{I}$, and we can proceed by a perturbative approach. In the limit of a tangential observation at the limb we have

$\frac{\mathrm{d}}{\mathrm{d} s} I\left(v, \boldsymbol{\Omega}_{\|}\right)=\frac{\mathrm{d}}{\mathrm{d} s} Q\left(v, \boldsymbol{\Omega}_{\|}\right)=0$,

and, after some algebra, we obtain (Trujillo Bueno 2003b; Landi Degl'Innocenti \& Landolfi 2004)

$\frac{Q}{I}=\frac{\varepsilon_{Q}\left(v, \mathbf{\Omega}_{\|}\right)}{\varepsilon_{I}\left(v, \mathbf{\Omega}_{\|}\right)}-\frac{\eta_{Q}\left(v, \mathbf{\Omega}_{\|}\right)}{\eta_{I}\left(v, \mathbf{\Omega}_{\|}\right)}$.

Since we are considering a two-term atom with unpolarized lower term, we have $\eta_{Q}=0$, and by substitution of Eq. (1) into Eq. (5), we obtain the relation

$\frac{Q}{I} \approx \frac{\varepsilon_{Q}}{\varepsilon_{I}}=\frac{3}{4} W_{2} w$

The quantity $\mathcal{W}_{2} \equiv W_{2}\left(L_{\ell} L_{\mathrm{u}} S, J_{\ell} J_{\mathrm{u}}\right)$, which is obtained from Eq. (2), setting $K=2$, is the generalization to the case of a twoterm atom of a similar quantity defined for a two-level atom, which is often referred to as "polarizability factor".

Equation (6) shows that, under the simplifying hypotheses introduced above, the fractional polarization is proportional to the product of the anisotropy factor and of the quantity $\mathcal{W}_{2}$, which depends on the quantum numbers of the transition. Figure 6 shows the values of $\mathcal{W}_{2}$ for a certain number of transitions. We can easily observe that the higher values, are found for transitions with $\Delta L=+1,0$, and with $\Delta J=\Delta L$, independently of the spin value, in agreement with the empirical results obtained from our analysis of the second solar spectrum.

Since we are neglecting radiation transfer effects, and since the theory we are applying neglects partial redistribution effects, we consider hereafter only the lines with small equivalent width, for which these effects should be small, or negligible. We consider therefore only the " $\mathrm{S}$ " signals (the corresponding lines have $W_{\lambda} / \lambda<30 \mathrm{~F}$, see Fig. 5, panel a), with the exception of the $\mathrm{H}_{\alpha}$ line (see Sect. 4.1). Recalling that the theory we are applying is based on the assumption that the atomic system is described by the $L-S$ coupling scheme, and recalling the hypothesis of the unpolarized lower term, we restrict from now on to consider only the "S" signals due to transitions between levels in $L-S$ coupling ${ }^{3}$, with a metastable (or fundamental) lower level.

Plotting the signal amplitude as a function of $\mathcal{W}_{2}$, we observe that all the points lie either close to the straight line passing through the origin, and having slope equal to 0.011 , or below it (see Fig. 5, panel d). According to Eq. (6), this behavior implies that the anisotropy factor $w$ has to be smaller than $(4 / 3) \times 0.011 \approx 0.015$. Since the anisotropy factor of the photospheric continuum, around $4000 \AA$, is of about 0.15 , we immediately notice that there is a discrepancy of about one order of magnitude ${ }^{4}$. Very likely, such a significant discrepancy is due to the fact that in Eq. (6) three fundamental "ingredients", all expected to decrease the polarization, are neglected: the magnetic field, collisions, and radiation transfer effects.

The effect of a unimodal microturbulent magnetic field can be taken into account rather easily. In the presence of such a magnetic field, in fact, Eq. (6) is modified into

$\frac{Q}{I} \approx \frac{3}{4} W_{2} w \frac{1}{5}\left(1+\frac{2}{1+H_{\mathrm{u}}^{2}}+\frac{2}{1+4 H_{\mathrm{u}}^{2}}\right)$,

\footnotetext{
3 As far as rare earth elements are concerned, if the percentage of the $L-S$ coupling of the upper or lower level is unknown, or smaller than $70 \%$ (see Table 2), the line has been discarded.

4 The value of 0.15 refers to the "solar surface", as defined in Sect. 12.3 of Landi Degl'Innocenti \& Landolfi (2004).
} 

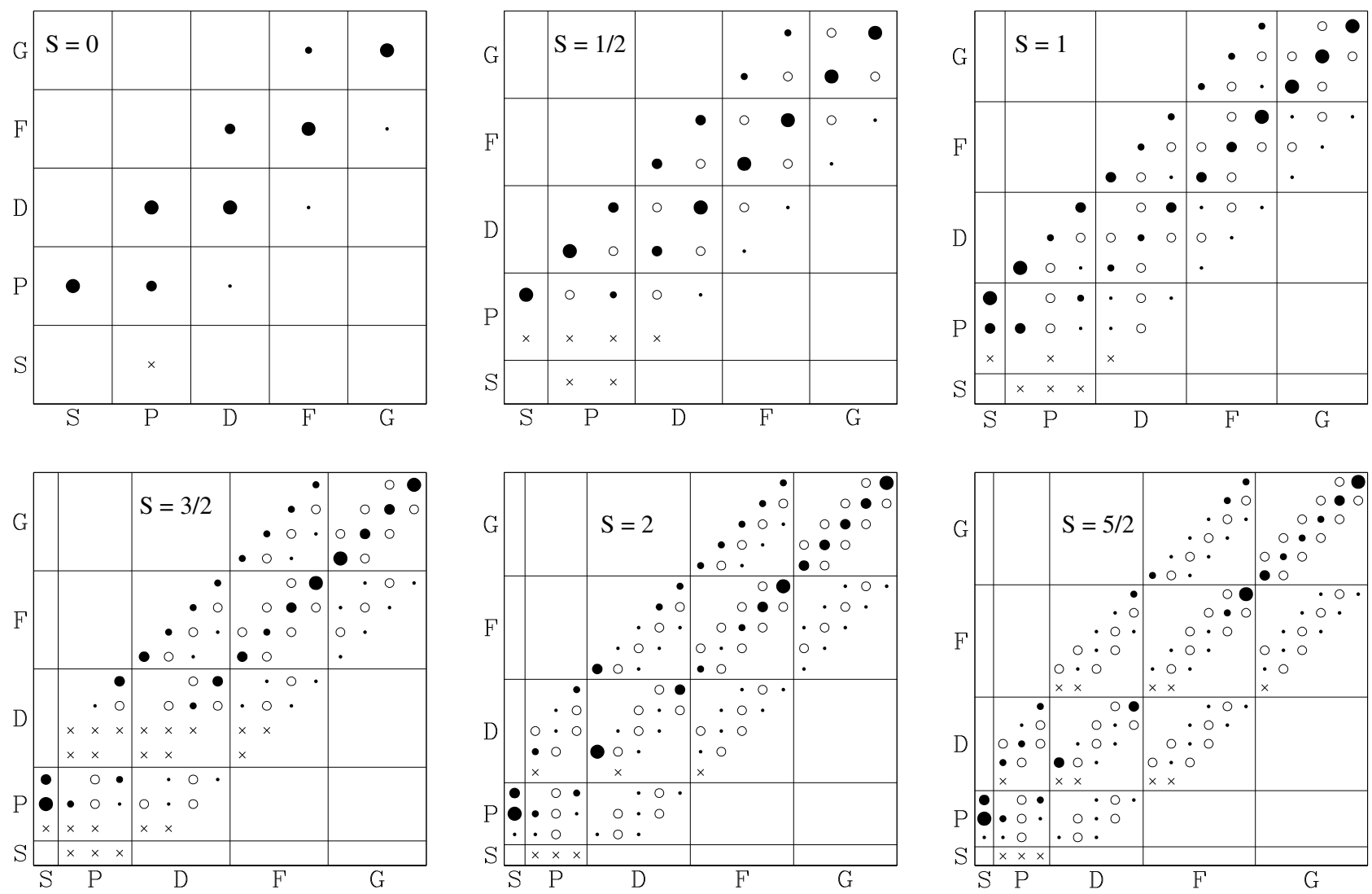

$\circ \mathrm{W}_{2} \leqq 0 \quad \times \mathrm{W}_{2}=0 \quad \cdot 0<\mathrm{W}_{2} \leqq 0.1 \quad \cdot 0.1<\mathrm{W}_{2}<0.2 \quad \bullet 0.2<\mathrm{W}_{2}<0.3 \quad \bullet \mathrm{W}_{2}>0.3$

Fig. 6. Values of $\mathcal{W}_{2}$ (see Eq. (2) for its definition) for different transitions. Each panel refers to a particular spin value. Within each panel the various boxes refer to a couple of values for $L$ ( $L_{\ell}$ on the horizontal axis, and $L_{\mathrm{u}}$ on the vertical axis). Within each box the points represent the allowed $J$ values of the transitions. The values of $J_{\ell}$ are reported in ascending order on the horizontal axis, the values of $J_{\mathrm{u}}$ are reported in ascending order on the vertical axis. Note that the highest values are found for transitions with $\Delta L=0,1$ (diagonal and upper-diagonal boxes), and $\Delta J=\Delta L$. This second property holds particularly for high $L$-values.

where $H_{\mathrm{u}}=B_{\mathrm{t}} / B_{\mathrm{c}}$, with $B_{\mathrm{t}}$ the microturbulent magnetic field intensity, and $B_{\mathrm{c}}$ the critical field intensity, defined (in Gauss) as

$B_{\mathrm{c}}=\frac{A}{0.879 g_{\mathrm{u}}}$

where $A$ is the Einstein coefficient for spontaneous emission, expressed in $10^{7} \mathrm{~s}^{-1}$, and $g_{\mathrm{u}}$ the Lande factor of the upper level ${ }^{5}$. The critical field represents therefore the magnetic field needed to decrease the polarization by about a factor 2 . Note that the critical field depends on the value of the Einstein coefficient, which in some cases is not known with high precision. Note also that two of the lines here considered (Ti I at $4536.054 \AA$, and $\mathrm{Cr}$ I at $3883.639 \AA$ ) have the Landé factor of the upper level equal to 0 , and therefore are not sensitive to the magnetic field. If we assume that a microturbulent magnetic field is present in the quiet sun, we expect that the observed polarization should be stronger in those lines having a larger critical field. In Fig. 5 panels $d-f$ the critical field intensity is represented through a color scale. The expected correlation between the polarization degree and the critical field intensity is suggested, although many "exceptions" are present. The role of the magnetic field is obviously important. However, it does not seem to be able to fully explain

\footnotetext{
${ }^{5}$ For the elements or ions showing hyperfine structure (HFS), the value of the Landé factor appearing in Eq. (8) is the one that would compete to the level neglecting HFS.
}

the discrepancy previously discussed. Setting the anisotropy factor equal to 0.015 (for all the lines), the best fit between the observed values of $Q / I$ and the values obtained through Eq. (7) is achieved for a microturbulent magnetic field on the order of 20 Gauss or larger (basically when most of the lines have reached the saturation regime). It is important to stress that this is nothing else than an exercise that can give some indications about the order of magnitude of the physical quantity under investigation. It would be naive to rely too much on the particular numerical value obtained, or even to give it a real physical meaning without keeping in mind the approximations that have been previously introduced. For a reliable inference of the strength of the magnetic field it is important to take into account the effects of radiative transfer and collisions in realistic models of the solar atmosphere, as shown by Trujillo Bueno et al. (2004) for the Sr I line at $4607 \AA$. Taking into account the effects of radiative transfer, and collisions for a large sample of lines is however much more difficult, also because our knowledge of the collisional rates is still very limited.

We conclude our discussion with two more plots based on Eq. (6). If we divide the observed value of $Q / I$ of each signal by the corresponding $W_{2}$, we should obtain something proportional to the anisotropy factor of the radiation field at the height of formation, and at the wavelength position of the various spectral lines. The anisotropy factor of the continuum has been plotted as a function of wavelength by 
Manso Sainz \& Landi Degl'Innocenti (2002), and it can be observed that in the wavelength range considered here, it decreases with wavelength approximately by a factor 2 . If we plot the quantity $R=(Q / I) / \mathcal{W}_{2}$ as a function of wavelength, we see that the points spread over the Cartesian plane, showing a slight decreasing trend with wavelength (see Fig. 5, panel e). The black dashed line, which represents the best linear fit to the points, shows more clearly the expected trend.

Since the observations of Gandorfer (Gandorfer 2000, 2002, 2005) were taken inside the limb, very likely the most relevant dependence that has to be considered is the variation of the anisotropy factor as a function of the height of formation of the various lines. In this sense it is therefore interesting to plot the quantity $R$ as a function of the reduced equivalent width, since, as a first approximation, lines with similar equivalent widths should form at similar heights. Note that the application of Eq. (6) for the investigation of this kind of dependence is not fully consistent, as it is correct only for tangential observations, and it is based upon the assumption that the illuminating radiation field should be the same for the various components of the multiplet. As can be observed from panel f of Fig. 5, in this case the points spread over the Cartesian plane, without showing any particular trend. As far as the sensitivity to a microturbulent magnetic field is concerned, we observe that, as expected, lines with larger critical fields are in general more polarized, but too many exceptions are present in order to draw any conclusion. On the contrary, these results stress the fact that the mechanism through which the polarization is produced, and modified, is extremely complex. In particular, there are many physical processes that are involved in the generation and modification of the polarization (see, for example, Shchukina \& Trujillo Bueno 2008) . As previously observed, for some of these processes we do not have a reliable knowledge of all the physical parameters which are needed in order to quantify their effects.

\section{Conclusions}

We have carried out a detailed analysis of the second solar spectrum, as plotted in graphical form in the three volumes of the atlas “The Second Solar Spectrum" (Gandorfer 2000, 2002, 2005). All the strongest polarization signals due to atomic transitions have been identified, classified according to the shape of their $Q / I$ profile, and listed together with the most relevant spectroscopic properties of the corresponding lines. From the analysis of these data, three empirical rules have been formulated. The rules concern the properties of the lower level, a correlation between the shape of the polarization profiles and the reduced equivalent width of the lines in the intensity spectrum, and the quantum numbers involved in the transitions. Our initial purpose to investigate the possible existence of common properties among the strongest polarization signals of the second solar spectrum has therefore been met with success. These three rules might represent the first step to understand why only particular spectral lines show at the limb strong polarization signals, which is one of the most interesting open questions concerning the second solar spectrum.

\section{References}

Brückner, G. 1963, ZAp, 58, 73

Casini, R., \& Landi Degl'Innocenti, E. 1994, A\&A, 291, 668

Fluri, D. M., \& Stenflo, J. O. 1999, A\&A, 341, 902

Gandorfer, A. 2000, The Second Solar Spectrum: A High Resolution Polarimetric Survey of Scattering Polarization at the Solar Limb in Graphical Representation, Vol. I: $4625 \AA$ to $6995 \AA$ (Zürich: vdf ETH), ISBN 37281 27647

Gandorfer, A. 2002, The Second Solar Spectrum: A High Resolution Polarimetric Survey of Scattering Polarization at the Solar Limb in Graphical Representation, Vol. II: $3910 \AA$ to $4630 \AA$ (Zürich: vdf ETH), ISBN 37281 28444

Gandorfer, A. 2005, The Second Solar Spectrum: A High Resolution Polarimetric Survey of Scattering Polarization at the Solar Limb in Graphical Representation, Vol. III: $3160 \AA$ to $3915 \AA ̊$ (Zürich: vdf ETH), ISBN 37281 30184

Kurucz, R. L., \& Bell, B. 1995, Atomic Line Data Kurucz CD-ROM No. 23 (Cambridge, Mass.: Smithsonian Astrophysical Observatory)

Landi Degl'Innocenti, E., \& Landolfi, M. 2004, Polarization in Spectral Lines (Dordrecht: Kluwer)

Manso Sainz, R., \& Landi Degl'Innocenti, E. 2002, A\&A, 394, 1093

Manso Sainz, R., \& Trujillo Bueno, J. 2003, A Multilevel Radiative Transfer Program for Modeling Scattering Line Polarization and the Hanle Effect in Stellar Atmospheres, in Solar Polarization 3, ed. J. Trujillo Bueno, \& J. Sánchez Almeida, ASP Conf. Ser., 307, 251

Manso Sainz, R., Landi Degl'Innocenti, E., \& Trujillo Bueno, J. 2006, A\&A, 447, 1125

Martin, W. C., Zalubas, R., \& Hagan, L. 1978, Atomic Energy Levels-The Rare Earth Elements, NSRDS-NBS 60 (Washington D.C.: NSRDS-NBS)

Moore, C. E. 1945, A Multiplet Table of Astrophysical Interest, Revised Edition, Part I-Table of Multiplets (New Jersey: Princeton)

Moore, C. E. 1949, Atomic Energy Levels As Derived From the Analyses of Optical Spectra. Vol. I (Washington D.C.: NBS)

Moore, C. E. 1958, Atomic Energy Levels As Derived From the Analyses of Optical Spectra. Vol. III (Washington D.C.: NBS)

Moore, C. E. 1971, Atomic Energy Levels As Derived From the Analyses of Optical Spectra. Vol. II, NSRDS-NBS 35 (Washington D.C.: NSRDS-NBS)

Moore, C. E., Minnaert, M. G. J., \& Houtgast, J. 1966, The Solar Spectrum $2935 \AA$ to $8770 \AA$, NBS Monograph 61 (Washington D.C.: NBS)

Ralchenko, Yu., Kramida, A. E., Reader, J., \& NIST ASD Team 2008, NIST Atomic Spectra Database (version 3.1.4) (Gaithersburg, MD.: NIST)

Redman, R. O. 1941, MNRAS, 101, 266

Shchukina, N., \& Trujillo Bueno, J. 2008, Three-dimensional Radiative Transfer Modeling of the Second Solar Spectrum of Titanium, in Solar Polarization 5, ed. S. Berdyugina, K. N. Nagendra, \& R. Ramelli, ASP Conf. Ser., in press

Stenflo, J. O. 1974, Sol. Phys., 37, 31

Stenflo, J. O. 1980, A\&A, 84, 68

Stenflo, J. O. 2005, A\&A, 429, 713

Stenflo, J. O., \& Keller, C. U. 1996, Nature, 382, 588

Stenflo, J. O., \& Keller, C. U. 1997, A\&A, 321, 927

Stenflo, J. O., Baur, T. G., \& Elmore, D. F. 1980, A\&A, 84, 60

Stenflo, J. O., Twerenbold, D., \& Harvey, J. W. 1983a, A\&AS, 52, 161

Stenflo, J. O., Twerenbold, D., Harvey, J. W., \& Brault, J. W. 1983b, A\&AS, 54 , 505

Stenflo, J. O., Keller, C. U., \& Gandorfer, A. 2000, A\&A, 355, 789

Trujillo Bueno, J. 2003a, The Generation and Transfer of Polarized Radiation in Stellar Atmospheres, in Stellar Atmosphere Modeling, ed. I. Hubeny, D. Mihalas, \& K. Werner, ASP Conf. Ser., 288, 551

Trujillo Bueno, J. 2003b, New Diagnostic Windows on the Weak Magnetism of the Solar Atmosphere, in Solar Polarization 3, ed. J. Trujillo Bueno, \& J. Sánchez Almeida, ASP Conf. Ser., 307, 407

Trujillo Bueno, J. 2009, The Magnetic Sensitivity of the Second Solar Spectrum, in Solar Polarization 5, ed. S. Berdyugina, K. N. Nagendra, \& R. Ramelli, ASP Conf. Ser., in press

Trujillo Bueno, J., Shchukina, N., \& Asensio Ramos, A. 2004, Nature, 430, 326 Wiehr, E. 1975, A\&A, 38, 303

Wiehr, E. 1978, A\&A, 67, 257 
L. Belluzzi and E. Landi Degl'Innocenti: Analysis of the most polarizing lines of the 2nd solar spectrum, Online Material $p 1$

Table 1. Table of the most polarizing atomic spectral lines of the second solar spectrum ( $3160 \AA-6995 \AA)$. The lines are listed by increasing wavelength. The various quantities that are listed for each line are described in Sect. 2.3.

\begin{tabular}{|c|c|c|c|c|c|c|c|c|c|}
\hline \# & $\begin{array}{l}\lambda^{\text {Sun }} \\
(\AA)\end{array}$ & Elem. & $\begin{array}{c}\mathrm{W}_{\lambda} \\
(\mathrm{m} \AA)\end{array}$ & $\begin{array}{c}\mathrm{W}_{\lambda} / \lambda \\
(\mathrm{F})\end{array}$ & $\begin{array}{c}(Q / I)^{\mathrm{M}} \\
(\%)\end{array}$ & $\begin{array}{c}(Q / I)^{\mathrm{m}} \\
(\%)\end{array}$ & $\begin{array}{l}Q / I \\
(\%)\end{array}$ & Type & Notes \\
\hline \multicolumn{10}{|c|}{$\longrightarrow$ Volume III -} \\
\hline 1 & 3179.342 & Ca II & 580 & 182 & 0.85 & 0.50 & 0.35 & W & 1 \\
\hline 2 & 3217.935 & Ti I & 35 & 11.1 & 0.75 & $0.60^{*}$ & 0.15 & $S^{*}$ & 2 \\
\hline 3 & 3230.592 & Sm II & 34 & 10.5 & 0.86 & $0.65^{*}$ & 0.21 & $\mathrm{~S}^{*}$ & 3 \\
\hline 4 & 3234.518 & Ti II & 49 & 15.2 & $\begin{array}{l}0.62 \\
1.35\end{array}$ & $\begin{array}{l}0.50 \\
0.85\end{array}$ & $\begin{array}{l}0.12 \\
0.50\end{array}$ & M1 & 4 \\
\hline 5 & 3242.007 & Ti II & 270 & 83.0 & $\begin{array}{l}0.95 \\
1.07\end{array}$ & $\begin{array}{l}0.80 \\
0.84\end{array}$ & $\begin{array}{l}0.15 \\
0.23\end{array}$ & M1 & \\
\hline 6 & 3247.569 & $\mathrm{Cu} \mathrm{I}$ & 246 & 76.0 & 1.75 & 0.82 & 0.93 & M0 & 5 \\
\hline 7 & 3248.612 & /Ti II Ti I & 148 & 46.0 & 0.57 & 0.40 & 0.17 & W & 6 \\
\hline 8 & 3260.552 & $\mathrm{Nb}$ II & 20 & 6.1 & 0.85 & $0.70^{*}$ & 0.15 & S & \\
\hline 9 & 3273.052 & $\mathrm{Zr}$ II & 78 & 23.9 & 0.77 & 0.58 & 0.19 & W & \\
\hline 10 & 3273.972 & $\mathrm{Cu} \mathrm{I}$ & $221^{*}$ & 67.4 & 0.36 & 0.19 & 0.17 & W & 7 \\
\hline 11 & 3308.399 & Ti I & 32 & 9.7 & 0.92 & $0.58^{*}$ & 0.34 & $\mathrm{~S}$ & \\
\hline 12 & 3322.949 & Ti II (Zr II) & $495^{*}$ & 157.3 & $\begin{array}{l}0.42 \\
0.75\end{array}$ & $\begin{array}{l}0.27 \\
0.64\end{array}$ & $\begin{array}{l}0.15 \\
0.11\end{array}$ & M1 & 8 \\
\hline 13 & 3326.777 & $/ / \mathrm{Ti}$ II-Zr II & 214 & 64.0 & 0.275 & 0.175 & 0.100 & W & \\
\hline 14 & 3372.812 & Ti II & 459 & 135.9 & 0.67 & 0.55 & 0.12 & M0 & \\
\hline 15 & 3380.260 & Ti II & 30 & 31.2 & 0.25 & 0.15 & 0.10 & $\mathrm{~W}$ & 9 \\
\hline 16 & 3380.585 & Ni I & 809 & 239 & 0.275 & 0.175 & 0.100 & W & 9 \\
\hline 17 & 3383.765 & Ti II & $430^{*}$ & 127 & 0.65 & 0.53 & 0.12 & M0 & \\
\hline 18 & 3391.973 & $\mathrm{Zr}$ II & $113^{*}$ & 20.0 & 0.42 & 0.32 & 0.10 & $\mathrm{~W}$ & \\
\hline 19 & 3405.126 & Co I & 206 & 60.5 & 0.67 & 0.49 & 0.18 & M0 & \\
\hline 20 & 3407.805 & Dy II & 60 & 18.8 & 0.42 & $0.32^{*}$ & 0.10 & $\mathrm{~S}^{*}$ & \\
\hline 21 & 3433.579 & $/ / \mathrm{Ni} \mathrm{I}-\mathrm{Cr} \mathrm{I}$ & 492 & 143 & 0.25 & 0.15 & 0.10 & W & \\
\hline 22 & 3439.229 & Gd II & 10 & 3.5 & 0.53 & $0.40^{*}$ & 0.13 & S & \\
\hline 23 & 3446.271 & Ni I & 470 & 136 & $\begin{array}{l}0.335 \\
0.575\end{array}$ & $\begin{array}{l}0.235 \\
0.435\end{array}$ & $\begin{array}{l}0.100 \\
0.14\end{array}$ & M1 & 10 \\
\hline 24 & 3452.905 & Ni I & 247 & 73.1 & 0.41 & 0.22 & 0.19 & W & \\
\hline 25 & 3453.512 & Co I & 310 & 87.9 & 0.36 & 0.24 & 0.12 & W & 11 \\
\hline
\end{tabular}

1 The linear polarization signal is quite noisy at this wavelength, and it is not possible to measure the amplitude of the $Q / I$ profile with high precision. There are four more lines in this spectral region that, although showing linear polarization peaks with amplitudes apparently larger than $0.1 \%$, have not been included in this table because of the high noise level: Fe I at $3171.665 \AA$, //Fe I at $3172.051 \AA$, Cr II Fe I p (CH) at $3172.087 \AA$, and Fe I at $3180.236 \AA$.

2 The polarization peak does not fall exactly at line center. This could be due to the fact that the line is severely blended with a depolarizing Ni I line at $3217.841 \AA$, which may imply also a reduction of the peak amplitude.

3 This signal is a typical case where it is not easy to establish the reference level from which the amplitude of the $Q / I$ peak has to be measured.

4 This signal has been associated with the Ti II line since the $Q / I$ central peak falls at the wavelength position of the line-core of this spectral line, and because it is very similar to the signal produced by the Ti II line at $3242.007 \AA$, which belongs to the same multiplet. However, the strong polarization signal in the wings may be produced by the combined action of this line and of the nearby line at $3234.647 \AA$ (Ni I-Fe I).

5 This is a very deep line in the intensity spectrum, and unavoidably the polarization signal is rather noisy at line center. For this reason it is not easy to determine if it has to be classified as "M0" or "M1". The polarization lobe in the blue wing of the line is almost completely destroyed by the presence of depolarizing blended lines.

6 The "W" shape of this signal might be due to the effect of depolarizing lines blended with the red and blue wings of this spectral line.

7 The polarization peak does not fall exactly at line center. This displacement, as well as the large width of the intensity profile of this line, could be explained by the presence of a blend with an $\mathrm{OH}$ line, or in terms of the hyperfine structure shown by this element.

8 The depolarization observed beside the line-core peak of this line might be affected by the presence of blended lines. Moreover, it is not possible to exclude that the polarization observed in the far wings could be affected (or due) to the NH band. In this latter case the signal should be classified as "W".

9 These lines are severely blended. The larger reduced equivalent width of the Ni I line suggests that the broad depolarization is due to this line, while the Ti II line produces a much narrower "W" signal.

10 The polarization peak at line center shows a substructure made of two secondary peaks separated by a small dip.

${ }^{11}$ It is not possible to exclude that this signal might be of type "M1", the wing-peaks being hidden by blended lines. 
Table 1. continued.

\begin{tabular}{|c|c|c|c|c|c|c|c|c|c|}
\hline$\#$ & $\begin{array}{l}\lambda^{\text {Sun }} \\
(\AA)\end{array}$ & Elem. & $\begin{array}{c}\mathrm{W}_{\lambda} \\
(\mathrm{m} \AA)\end{array}$ & $\begin{array}{c}\mathrm{W}_{\lambda} / \lambda \\
(\mathrm{F})\end{array}$ & $\begin{array}{c}(Q / I)^{\mathrm{M}} \\
(\%)\end{array}$ & $\begin{array}{c}(Q / I)^{\mathrm{m}} \\
(\%)\end{array}$ & $\begin{array}{l}Q / I \\
(\%)\end{array}$ & Type & Notes \\
\hline 26 & 3458.467 & Ni I & 656 & 189 & $\begin{array}{l}0.28 \\
0.60\end{array}$ & $\begin{array}{l}0.14 \\
0.42\end{array}$ & $\begin{array}{l}0.14 \\
0.18\end{array}$ & M1 & \\
\hline 27 & 3461.667 & Ni I & 758 & 219 & 0.33 & 0.16 & 0.17 & W & 10 \\
\hline 28 & 3464.474 & $\mathrm{Sr}$ II (Fe II) & 63 & 18.2 & 0.44 & $0.32^{*}$ & 0.12 & S & \\
\hline 29 & 3465.766 & Co I & 69 & 84.2 & 0.240 & 0.125 & 0.115 & W & 12 \\
\hline 30 & 3465.880 & $\mathrm{Fe} \mathrm{I}$ & 544 & 158 & 0.26 & 0.15 & 0.11 & W & 12 \\
\hline 31 & 3469.493 & Ni I (V II) & 180 & 52.2 & 0.27 & 0.15 & 0.12 & W & \\
\hline 32 & 3472.558 & Ni I & 374 & 136 & 0.31 & 0.175 & 0.135 & W & \\
\hline 33 & 3476.712 & $\mathrm{Fe} \mathrm{I}$ & 465 & 136 & 0.34 & 0.19 & 0.15 & W & \\
\hline 34 & 3485.110 & $\mathrm{Ni} \mathrm{I}$ & 100 & 29.2 & 0.33 & $0.23^{*}$ & 0.10 & $\mathrm{~S}^{*}$ & \\
\hline 35 & 3492.975 & Ni I & 826 & 239 & 0.25 & 0.15 & 0.10 & W & \\
\hline 36 & 3494.515 & /Cr II Dy II & 46 & 13.2 & 0.375 & $0.26^{*}$ & 0.115 & $\mathrm{~S}^{*}$ & 13 \\
\hline 37 & 3504.892 & $\mathrm{Fe}$ I /Ti II & 132 & 37.6 & 0.36 & 0.22 & 0.14 & W & \\
\hline 38 & 3510.846 & Ti II & 87 & 29.1 & 0.40 & $0.28^{*}$ & 0.12 & $\mathrm{~S}$ & \\
\hline 39 & 3515.066 & $\mathrm{Ni} \mathrm{I}$ & 718 & 202 & 0.22 & 0.10 & 0.12 & W & \\
\hline 40 & 3519.618 & $\mathrm{Zr} \mathrm{I}$ & 7.5 & 2.6 & 0.36 & $0.23^{*}$ & 0.13 & $\mathrm{~S}$ & \\
\hline 41 & 3521.270 & $\mathrm{Fe} \mathrm{I}$ & 381 & 110 & 0.24 & 0.11 & 0.13 & W & \\
\hline 42 & 3533.014 & $\mathrm{Fe} \mathrm{I}$ & 97 & 31.4 & 0.35 & 0.20 & 0.15 & W & 14 \\
\hline 43 & 3550.222 & -Dy II & 43 & 12.0 & 0.42 & 0.31 & 0.11 & S & 15 \\
\hline 44 & 3553.095 & Pd I & $48^{*}$ & 3.4 & 0.385 & $0.20^{*}$ & 0.185 & $S$ & 16 \\
\hline 45 & 3554.937 & $\mathrm{Fe} \mathrm{I}$ & 404 & 111 & 0.21 & 0.11 & 0.10 & W & \\
\hline 46 & 3556.597 & Zr II & 23 & 7.0 & 0.32 & $0.18^{*}$ & 0.14 & S & \\
\hline 47 & 3558.532 & $/ \mathrm{Fe} \mathrm{I}-\mathrm{Sc}$ II & 485 & 137 & 0.23 & 0.05 & 0.18 & W & \\
\hline 48 & 3565.396 & $\mathrm{Fe} \mathrm{I}$ & 990 & 274 & 0.20 & 0.08 & 0.12 & W & 17 \\
\hline 49 & 3566.383 & $\mathrm{Ni} \mathrm{I}$ & 458 & 141 & $\begin{array}{l}0.32 \\
0.38\end{array}$ & $\begin{array}{l}0.22 \\
0.30\end{array}$ & $\begin{array}{l}0.10 \\
0.08\end{array}$ & M1 & 17 \\
\hline 50 & 3570.134 & $\mathrm{Fe} \mathrm{I}$ & 1380 & 387 & $\begin{array}{l}0.34 \\
0.41\end{array}$ & $\begin{array}{l}0.20 \\
0.29\end{array}$ & $\begin{array}{l}0.14 \\
0.12\end{array}$ & M1 & \\
\hline 51 & 3578.693 & Cr I & 488 & 142 & $\begin{array}{l}0.42 \\
0.435\end{array}$ & $\begin{array}{l}0.17 \\
0.285\end{array}$ & $\begin{array}{l}0.25 \\
0.150\end{array}$ & M1 & \\
\hline 52 & 3581.209 & $\mathrm{Fe} \mathrm{I}$ & 2144 & 599 & $\begin{array}{l}0.34 \\
0.41\end{array}$ & $\begin{array}{l}0.18 \\
0.28\end{array}$ & $\begin{array}{l}0.16 \\
0.13\end{array}$ & M1 & \\
\hline 53 & 3585.714 & $\mathrm{Fe} \mathrm{I}$ & 168 & 63.0 & 0.22 & 0.11 & 0.11 & W & 18 \\
\hline 54 & 3585.844 & Co I?-Ti I & 25 & 22.6 & 0.40 & $0.26^{*}$ & 0.14 & S & 18 \\
\hline 55 & 3586.990 & $\mathrm{Fe} \mathrm{I}$ & 532 & 147 & 0.22 & 0.09 & 0.13 & W & \\
\hline 56 & 3589.222 & $\mathrm{Ru} I$ & 18 & 5.7 & 0.375 & 0.275 & 0.100 & S & \\
\hline 57 & 3590.489 & Sc II & 136 & 37.9 & $\begin{array}{l}0.21 \\
0.375\end{array}$ & $\begin{array}{l}0.05 \\
0.275\end{array}$ & $\begin{array}{l}0.16 \\
0.100\end{array}$ & M1 & 19 \\
\hline
\end{tabular}

12 These lines are severely blended, and it is difficult to understand if they are both "W", or if only one is "W", the other one being "S".

13 The polarization peak does not fall exactly at line center.

14 This line falls in the blue depolarizing wing of a Fe I line, and it is not easy to determine if this signal has to be classified as "S" or "W".

15 The polarization peak has quite a large width. This could be due to the unidentified blended line, or to the hyperfine structure shown by this element. This second hypothesis is supported by the similarity with the polarization signal of the Dy II line at $3407.805 \AA$.

${ }^{16}$ It is not easy to establish the reference level from which the amplitude of the $Q / I$ peak has to be measured.

17 It is not possible to exclude that the polarization peak at $3566.7 \AA$ could be due to the interferences between the Fe I lines at $3565.396 \AA$ and $3570.134 \AA$, which belong to the same multiplet. In this case the Fe I signal should be classified as "M1" instead of "W", while the Ni I signal as "W" instead of "M1".

18 The width of the polarization peak at the wavelength position of the Co I-Ti I line (line \# 54) is a little surprising. It is not possible to exclude that this peak might pertain to a possible "M1" structure of the Fe I line at $3585.714 \AA$. In this case, the Co I-Ti I line should be excluded.

${ }^{19}$ In the blue wing of this line the polarization might be enhanced by (or due to) the presence of a blending Fe I line at $3590.302 \AA$, in the red wing it could be affected by the wing-lobe of the far, strong $\mathrm{Cr}$ I line at $3593.495 \AA$. It is difficult, therefore, to determine if this line should be classified as "M1", or "W". 
Table 1. continued.

\begin{tabular}{|c|c|c|c|c|c|c|c|c|c|}
\hline \# & $\begin{array}{l}\lambda_{\text {Sun }} \\
(\AA)\end{array}$ & Elem. & $\begin{array}{c}\mathrm{W}_{\lambda} \\
(\mathrm{m} \AA)\end{array}$ & $\begin{array}{c}\mathrm{W}_{\lambda} / \lambda \\
(\mathrm{F})\end{array}$ & $\begin{array}{c}(Q / I)^{\mathrm{M}} \\
(\%)\end{array}$ & $\begin{array}{c}(Q / I)^{\mathrm{m}} \\
(\%)\end{array}$ & $\begin{array}{l}Q / I \\
(\%)\end{array}$ & Type & Notes \\
\hline 58 & 3592.027 & V II & 75 & 21.0 & 0.20 & 0.10 & 0.10 & W & \\
\hline 59 & 3593.495 & $\mathrm{Cr} \mathrm{I}$ & 436 & 127 & $\begin{array}{l}0.775 \\
0.95\end{array}$ & $\begin{array}{l}0.270 \\
0.27\end{array}$ & $\begin{array}{l}0.505 \\
0.68\end{array}$ & M1 & \\
\hline 60 & 3594.638 & $\mathrm{Fe} \mathrm{I}$ & 146 & 40.8 & 0.30 & 0.17 & 0.13 & W & \\
\hline 61 & 3594.876 & Co I & 92 & 25.7 & 0.25 & 0.14 & 0.11 & $\mathrm{~W}$ & \\
\hline 62 & 3596.054 & Ti II & 95 & 26.7 & 0.21 & 0.07 & 0.14 & W & 20 \\
\hline 63 & 3597.712 & $\mathrm{Ni} I$ & 181 & 50.2 & $\begin{array}{l}0.29 \\
0.36\end{array}$ & $\begin{array}{l}0.12 \\
0.27\end{array}$ & $\begin{array}{l}0.17 \\
0.09\end{array}$ & M1 & 21 \\
\hline 64 & 3600.739 & Y II & 69 & 19.2 & 0.35 & 0.25 & 0.10 & W & 22 \\
\hline 65 & 3601.198 & $\mathrm{Zr} \mathrm{I}$ & 13 & 3.6 & 0.42 & $0.31^{*}$ & 0.11 & $\mathrm{~S}$ & \\
\hline 66 & 3602.287 & $\mathrm{Ni} I$ & 94 & 26.2 & 0.18 & 0.06 & 0.12 & $\mathrm{~W}$ & \\
\hline 67 & 3605.339 & $/ / \mathrm{Cr}$ I Co I & 495 & 136 & 0.24 & 0.09 & 0.15 & W & \\
\hline 68 & 3608.869 & $\mathrm{Fe} \mathrm{I}$ & 1046 & 287 & $\begin{array}{l}0.31 \\
0.44\end{array}$ & $\begin{array}{l}0.18 \\
0.26\end{array}$ & $\begin{array}{l}0.13 \\
0.18\end{array}$ & M1 & \\
\hline 69 & 3610.166 & $/ / \mathrm{Fe}$ I Ti I & 231 & 65.4 & 0.26 & 0.11 & 0.15 & W & \\
\hline 70 & 3610.460 & Ni I & $250^{*}$ & 52.0 & 0.27 & 0.08 & 0.19 & W & \\
\hline 71 & 3618.777 & $\mathrm{Fe} \mathrm{I}$ & 1410 & 385 & $\begin{array}{l}0.39 \\
0.35\end{array}$ & $\begin{array}{l}0.15 \\
0.25\end{array}$ & $\begin{array}{l}0.24 \\
0.10\end{array}$ & M1 & 23 \\
\hline 72 & 3619.400 & Ni I & 568 & 204 & 0.30 & 0.16 & 0.14 & W & \\
\hline 73 & 3621.467 & $\mathrm{Fe} \mathrm{I}$ & 140 & 40.6 & 0.19 & 0.09 & 0.10 & W & \\
\hline 74 & 3624.733 & $\mathrm{Ni} \mathrm{I}$ & 132 & 37.1 & 0.165 & 0.04 & 0.125 & W & \\
\hline 75 & 3631.475 & $/ / \mathrm{Fe}$ I Cr II & 1364 & 369 & $\begin{array}{l}0.21 \\
0.31\end{array}$ & $\begin{array}{l}0.11 \\
0.24\end{array}$ & $\begin{array}{l}0.10 \\
0.07\end{array}$ & M1 & \\
\hline 76 & 3633.138 & Y II & $134^{*}$ & 19.0 & 0.51 & 0.24 & 0.27 & S & \\
\hline 77 & 3647.851 & $\mathrm{Fe} \mathrm{I}(\mathrm{Fe} \mathrm{I})$ & 970 & 313 & $\begin{array}{l}0.28 \\
0.31\end{array}$ & $\begin{array}{l}0.11 \\
0.23\end{array}$ & $\begin{array}{l}0.17 \\
0.08\end{array}$ & M1 & \\
\hline 78 & 3651.800 & Sc II & 114 & 39.1 & 0.14 & 0.04 & 0.10 & W & \\
\hline 79 & 3664.097 & Ni I & 130 & 43.8 & 0.19 & 0.08 & 0.11 & W & \\
\hline 80 & 3674.150 & Ni I & $178^{*}$ & 33.0 & 0.16 & 0.06 & 0.10 & W & \\
\hline 81 & 3694.817 & Dy II & 24 & 9.2 & 0.30 & $0.20^{*}$ & 0.10 & $\mathrm{~S}$ & \\
\hline 82 & 3705.577 & $\mathrm{Fe} \mathrm{I}$ & 562 & 180 & 0.15 & 0.05 & 0.10 & $\mathrm{~W}$ & \\
\hline 83 & 3710.292 & Y II & 74 & 27.2 & 0.40 & $0.25^{*}$ & 0.15 & $\mathrm{~S}$ & \\
\hline 84 & 3719.947 & $\mathrm{Fe} \mathrm{I}$ & 1664 & 538 & 0.34 & 0.24 & 0.10 & M0 & 24 \\
\hline 85 & 3724.574 & Ti I & 60 & 23.1 & 0.475 & 0.320 & 0.155 & $\mathrm{~S}$ & \\
\hline 86 & 3728.042 & $\mathrm{Ru} \mathrm{I}$ & 40 & 20.0 & 0.30 & $0.20^{*}$ & 0.10 & $\mathrm{~S}^{*}$ & 25 \\
\hline 87 & 3733.841 & Cr I? & 2 & 2.3 & 0.57 & 0.42 & 0.15 & S & 26 \\
\hline 88 & 3734.874 & $\mathrm{Fe} \mathrm{I}$ & 3027 & 945 & 0.480 & 0.275 & 0.205 & W & 27 \\
\hline 89 & 3737.141 & $\mathrm{Fe} \mathrm{I}$ & 1071 & 428 & 0.31 & 0.16 & 0.15 & W & \\
\hline 90 & 3741.312 & Sm II-Eu II & 13 & 5.0 & 0.57 & $0.38^{*}$ & 0.19 & $\mathrm{~S}$ & 28 \\
\hline 91 & 3742.278 & Ru I & 6 & 2.4 & 0.50 & $0.375^{*}$ & 0.125 & $\mathrm{~S}$ & \\
\hline
\end{tabular}

${ }^{20}$ Very likely the polarization in the blue wing is due to the strong $\mathrm{Cr}$ I line at $3593.495 \AA$, while in the red wing to the Ni I line at $3597.712 \AA$. If this is not the case, the signal should be classified as "M1".

${ }^{21}$ It is not possible to exclude that the polarization in the far wings of this line might be the wing-lobes of the far Cr I line at $3593.495 \AA$. In this case the signal should be classified as "W".

22 The polarization lobes in the far wings do not seem to be due to this line. If this is not the case, the signal should be classified as "M1".

23 We have classified this signal as "M1", since its wing-lobes appear to be severely blended by several depolarizing lines.

${ }^{24}$ This line produces a very broad polarization signal. The amplitude of the wing-lobes has been measured in the blue wing at $3717.0 \AA$.

25 The polarization peak does not fall exactly at line center, being slightly shifted to the blue.

26 This polarization signal, though being located at the wavelength position corresponding to a (possible) $\mathrm{Cr}$ I line, might be due either to $\mathrm{CN}$ or to the strong, nearby Fe I line at $3734.874 \AA$.

27 It is not possible to exclude that the signal at $3733.841 \AA$ (\# 87) might be due to this Fe I line. In this case this signal should be classified as "M1" instead of "W".

28 The polarization peak does not fall exactly at line center, being slightly shifted to the blue. This may indicate that the signal is due to Sm II. 
Table 1. continued.

\begin{tabular}{|c|c|c|c|c|c|c|c|c|c|}
\hline$\#$ & $\begin{array}{l}\lambda^{\text {Sun }} \\
(\AA)\end{array}$ & Elem. & $\begin{array}{c}\mathrm{W}_{\lambda} \\
(\mathrm{m} \AA)\end{array}$ & $\begin{array}{c}\mathrm{W}_{\lambda} / \lambda \\
(\mathrm{F})\end{array}$ & $\begin{array}{c}(Q / I)^{\mathrm{M}} \\
(\%)\end{array}$ & $\begin{array}{c}(Q / I)^{\mathrm{m}} \\
(\%)\end{array}$ & $\begin{array}{l}Q / I \\
(\%)\end{array}$ & Type & Notes \\
\hline 92 & 3743.368 & $\mathrm{Fe} \mathrm{I}$ & 592 & 193 & 0.275 & 0.175 & 0.100 & W & \\
\hline 93 & 3745.574 & $\mathrm{Fe} \mathrm{I}$ & $1202^{*}$ & 459 & 0.325 & 0.200 & 0.125 & W & \\
\hline 94 & 3745.910 & Fe I (Zr II) & 540 & 301 & 0.575 & $0.280^{*}$ & 0.295 & $\mathrm{~S}$ & 29 \\
\hline 95 & 3748.271 & $\mathrm{Fe} \mathrm{I}$ & 497 & 228 & 0.32 & 0.18 & 0.14 & W & \\
\hline 96 & 3748.966 & $/ \mathrm{Fe} \mathrm{I} \mathrm{Cr} \mathrm{I}$ & 103 & 82.9 & 0.275 & 0.175 & 0.100 & W & \\
\hline 97 & 3749.495 & $\mathrm{Fe} \mathrm{I}$ & 1907 & 578 & 0.45 & 0.20 & 0.25 & W & \\
\hline 98 & 3753.620 & $/ \mathrm{Fe} \mathrm{I}-\mathrm{Ti} \mathrm{I}$ & 132 & 45.0 & 0.16 & 0.30 & 0.14 & W & 30 \\
\hline 99 & 3758.245 & Fe I & 1647 & 497 & $\begin{array}{l}0.40 \\
0.69\end{array}$ & $\begin{array}{l}0.22 \\
0.42\end{array}$ & $\begin{array}{l}0.18 \\
0.27\end{array}$ & M1 & \\
\hline 100 & 3759.299 & Ti II & 334 & 115 & 0.41 & 0.31 & 0.10 & W & 31 \\
\hline 101 & 3761.320 & Ti II & $277^{*}$ & 61.0 & $\begin{array}{l}0.48 \\
0.70\end{array}$ & $\begin{array}{l}0.38 \\
0.41\end{array}$ & $\begin{array}{l}0.10 \\
0.29\end{array}$ & M1 & \\
\hline 102 & 3763.803 & $\mathrm{Fe} \mathrm{I}$ & 829 & 255 & $\begin{array}{l}0.36 \\
0.72\end{array}$ & $\begin{array}{l}0.15 \\
0.40\end{array}$ & $\begin{array}{l}0.21 \\
0.32\end{array}$ & M1 & 32 \\
\hline 103 & 3767.204 & $\mathrm{Fe} \mathrm{I}$ & 820 & 262 & $\begin{array}{l}0.28 \\
0.55\end{array}$ & $\begin{array}{l}0.18 \\
0.40\end{array}$ & $\begin{array}{l}0.10 \\
0.15\end{array}$ & M1 & \\
\hline 104 & 3780.516 & $/ \mathrm{CN}-\mathrm{Zr} \mathrm{I}$ & 26 & 9.5 & 0.51 & 0.39 & 0.12 & S & 33 \\
\hline 105 & 3782.318 & CN/Y II & 18 & 6.3 & 0.485 & 0.385 & 0.100 & S & \\
\hline 106 & 3784.252 & Nd II-Fe I p & 16 & 5.8 & 0.65 & 0.38 & 0.27 & S & 34 \\
\hline 107 & 3787.236 & V II CN & $139^{*}$ & 17.2 & 0.50 & 0.38 & 0.12 & $S$ & \\
\hline 108 & 3788.861 & $/ \mathrm{Cr} \mathrm{I}-\mathrm{CN}$ & $69^{*}$ & 17.4 & 0.59 & 0.38 & 0.21 & S & \\
\hline 109 & 3791.194 & CN Nb I & 8.5 & 3.2 & 0.50 & 0.38 & 0.12 & $S$ & \\
\hline 110 & 3792.565 & /CN Y II & 33 & 15.0 & 0.67 & 0.38 & 0.29 & $S$ & \\
\hline 111 & 3796.974 & CN Cr I & 20 & 16.1 & 0.540 & 0.375 & 0.165 & S & \\
\hline 112 & 3801.371 & $\mathrm{Fe} \mathrm{I}-\mathrm{CN}$ & 75 & 30.2 & 0.550 & 0.375 & 0.175 & S & \\
\hline 113 & 3801.542 & $\mathrm{Ce}$ II CN & 50 & 21.0 & 0.575 & 0.375 & 0.200 & S & \\
\hline 114 & 3806.375 & $\mathrm{CN} F e \mathrm{I}$ & 67 & 22.0 & 0.63 & 0.37 & 0.26 & S & \\
\hline 115 & 3807.937 & $/ \mathrm{Cr} \mathrm{I}-\mathrm{CN}$ & 111 & 35.2 & 0.52 & $0.39^{*}$ & 0.13 & S & 35 \\
\hline 116 & 3809.162 & $\mathrm{Fe} \mathrm{I}$ & 24 & 9.5 & 0.62 & 0.37 & 0.25 & S & 36 \\
\hline 117 & 3811.380 & CN Ti I? & 35 & 16.0 & 0.64 & 0.37 & 0.27 & S & \\
\hline 118 & 3812.199 & CN Y II & $44^{*}$ & 4.5 & 0.51 & 0.37 & 0.14 & S & \\
\hline 119 & 3813.491 & V I & 54 & 28.4 & 0.49 & 0.36 & 0.13 & S & 37 \\
\hline 120 & 3814.892 & CN Ti I & 26 & 15.7 & 0.58 & 0.36 & 0.22 & S & \\
\hline 121 & 3817.843 & V I CN Cr I & 40 & 16.0 & 0.50 & 0.36 & 0.14 & $\mathrm{~S}$ & \\
\hline 122 & 3824.750 & $\mathrm{CN}$ Fe I p & 11 & 10.5 & 0.31 & $0.20^{*}$ & 0.11 & $\mathrm{~S}^{*}$ & \\
\hline 123 & 3826.770 & $\mathrm{CN}-\mathrm{V}$ I Ni I & 15 & 11.2 & 0.48 & 0.35 & 0.13 & $\mathrm{~S}$ & \\
\hline 124 & 3826.957 & $\mathrm{CN}-/ \mathrm{V}$ II & 32 & 21.7 & 0.47 & 0.35 & 0.12 & S & \\
\hline
\end{tabular}

${ }^{29}$ This signal falls in the depolarizing wing of the broad Fe I line at $3745.574 \AA$ (belonging to the same multiplet), and it is difficult to determine if this is a "S" or a "W" signal.

30 The dip in the red wing, which is free of blends, induces to believe that this is a "W" signal, the dip in the blue wing being missing because of the blend with the Dy II-CN line at $3753.525 \AA$.

31 This signal falls in the strongly polarized red wing of the Fe I line at $3758.245 \AA$, and it is not possible to exclude that it might be classified as an "M" signal. Note that the Ti II line at $3761.320 \AA$ (classified as "M1") belongs to the same multiplet.

32 The amplitude of the wing-lobe might be enhanced by the presence of $\mathrm{CN}$ lines.

33 The polarization peak does not fall exactly at line center, but it is slightly shifted to the blue. This may be an indication that the signal is due to $\mathrm{CN}$.

${ }^{34}$ Given the extremely low value of the Einstein coefficient of the Fe I line $\left(2 \times 10^{3} \mathrm{~s}^{-1}\right)$, it seems quite reasonable to believe that this signal is produced by the Nd II line.

35 The polarization peak does not fall exactly at line center, but it is slightly red-shifted. This is a strong hint that this signal might be due to CN.

36 The polarization peak does not seem to be as sharp as for an isolated line. Probably there is a contribution coming from the CN molecule.

37 The width of this polarization signal suggests that it might be enhanced by (or due to) the strongly blending Ti II line at $3813.394 \AA$. It is not possible to exclude therefore that the Ti II line should be included in the table, and classified as "M0". 
Table 1. continued.

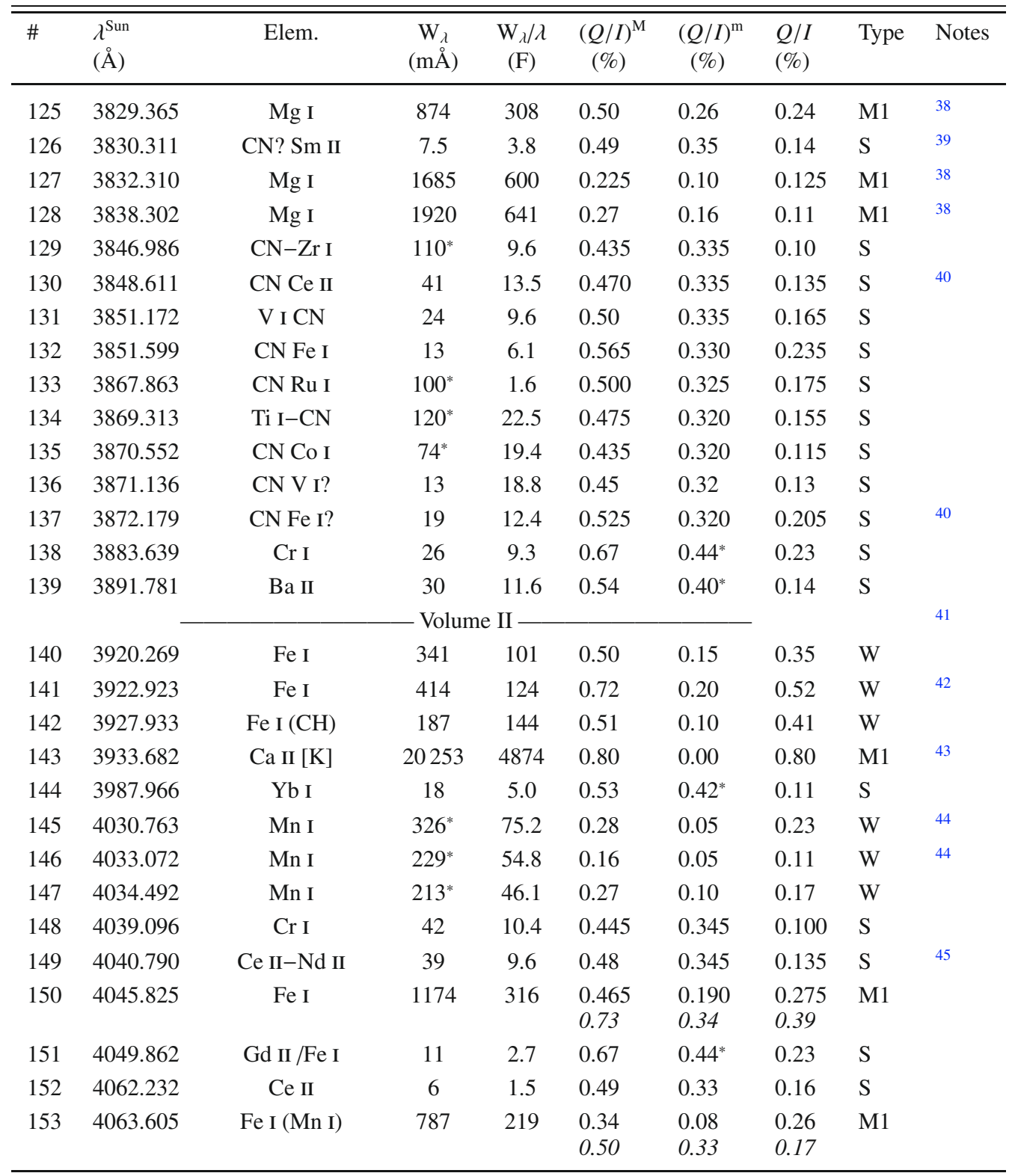

38 The wing-lobes of these lines are probably heavily affected (enhanced) by several CN lines. For this reason the wing-lobe amplitudes have not been measured.

39 The polarization signal is severely blended with other $\mathrm{CN}$ lines.

40 The polarization peak does not fall exactly at line center, but is somewhat red-shifted.

41 Volume III of the atlas covers the spectral interval between $3160 \AA$ and $3915 \AA$, Vol. II the spectral interval between $3910 \AA$ and $4630 \AA$. The spectral interval between $3910.0 \AA$ and $3913.5 \AA$ is covered by both volumes. Comparing the observations made in this spectral interval, it can be noticed that the theoretical continuum plotted in Vol. III is slightly lower than in Vol. II, while the peaks observed in $Q / I$ in Vol. III are slightly larger than in Vol. II. This might be due to the fact that, although the nominal position of the slit is $\mu=0.1$ for the observations of both atlases, because of the seeing the "effective" position might be different from the nominal value.

42 At line center this signal shows quite a complex profile, with several secondary peaks and dips.

43 At line center the Ca II K line shows a "M" type profile, but it is important to note that quantum interferences between the Ca II H and $\mathrm{K}$ lines produce a much broader signal that starts in the far blue wing of the $\mathrm{K}$ line, at about $3890 \AA$, with a positive lobe. The polarization becomes negative between the $\mathrm{K}$ and $\mathrm{H}$ lines, and it is zero (without showing any particular structure) at the wavelength position of the $\mathrm{H}$ line. This structure (which does not enter the classification scheme proposed in this work) has already been explained in terms of quantum interferences by Stenflo (1980) and Landi Degl'Innocenti \& Landolfi (2004). It is quite surprising, however, that the atlas does not show any positive lobe in the red wing of the $\mathrm{Ca}$ II $\mathrm{H}$ line, predicted on the other hand by the theory. This might be due to the choice of the level of the local polarized continuum, which may be wrong between $3972 \AA$ and $3990 \AA$. Note that where the polarization is negative, a depolarizing line produces a $Q / I$ peak. For this reason, all the lines producing polarization peaks in this spectral interval have not been included in the table. Note also that three lines (Sc I, V I and Co I), falling within an interval of $\approx 0.5 \AA$ centered at the core of the Ca II K line, are listed as masked lines by Moore et al. (1966).

44 At line center this signal shows quite a complex profile with several secondary peaks and dips, perhaps due to hyperfine structure.

45 The polarization signal is slightly blue-shifted with respect to line center. 
Table 1. continued.

\begin{tabular}{|c|c|c|c|c|c|c|c|c|c|}
\hline$\#$ & $\begin{array}{l}\lambda^{\text {Sun }} \\
(\AA)\end{array}$ & Elem. & $\begin{array}{c}\mathrm{W}_{\lambda} \\
(\mathrm{m \AA})\end{array}$ & $\begin{array}{c}\mathrm{W}_{\lambda} / \lambda \\
(\mathrm{F})\end{array}$ & $\begin{array}{c}(Q / I)^{\mathrm{M}} \\
(\%)\end{array}$ & $\begin{array}{c}(Q / I)^{\mathrm{m}} \\
(\%)\end{array}$ & $\begin{array}{l}Q / I \\
(\%)\end{array}$ & Type & Notes \\
\hline 154 & 4071.749 & $\mathrm{Fe} \mathrm{I}$ & 723 & 191 & $\begin{array}{l}0.41 \\
0.63\end{array}$ & $\begin{array}{l}0.16 \\
0.32\end{array}$ & $\begin{array}{l}0.25 \\
0.31\end{array}$ & M1 & 46 \\
\hline 155 & 4077.724 & Sr II & $428^{*}$ & 100 & $\begin{array}{l}1.225 \\
1.10\end{array}$ & $\begin{array}{l}0.320 \\
0.32\end{array}$ & $\begin{array}{l}0.905 \\
0.78\end{array}$ & MS & \\
\hline 156 & 4083.226 & Ce II & 26 & 6.4 & 0.47 & 0.32 & 0.15 & S & \\
\hline 157 & 4109.450 & Nd II & 39 & 9.5 & 0.42 & 0.30 & 0.12 & S & \\
\hline 158 & 4115.376 & Ce II & 11 & 2.7 & 0.430 & 0.295 & 0.135 & S & \\
\hline 159 & 4128.309 & Y I & 12 & 2.9 & 0.595 & 0.290 & 0.305 & S & \\
\hline 160 & 4129.724 & $\mathrm{Eu}$ II & 54 & 12.1 & 0.465 & 0.285 & 0.180 & S & \\
\hline 161 & 4130.657 & Ba II & 45 & 10.9 & 0.53 & $0.26^{*}$ & 0.27 & S & 47 \\
\hline 162 & 4142.842 & Y I & 22 & 5.3 & 0.445 & 0.280 & 0.165 & S & \\
\hline 163 & 4186.126 & Ti I & 42 & 10.0 & 0.53 & $0.27^{*}$ & 0.26 & S & \\
\hline 164 & 4222.602 & Ce II & 22 & 5.2 & 0.42 & $0.30^{*}$ & 0.12 & S & \\
\hline 165 & 4226.740 & $\mathrm{Ca} \mathrm{I}$ & 1476 & 342 & $\begin{array}{l}2.190 \\
2.700\end{array}$ & $\begin{array}{l}0.235 \\
0.235\end{array}$ & $\begin{array}{l}1.955 \\
2.465\end{array}$ & M1 & \\
\hline 166 & 4233.612 & $\mathrm{Fe} \mathrm{I}$ & 298 & 70.4 & 0.14 & 0.24 & 0.10 & W & 48 \\
\hline 167 & 4235.949 & $\mathrm{Fe} \mathrm{I}(\mathrm{Cr} \mathrm{I})(\mathrm{Y} \mathrm{I})$ & 385 & 90.9 & 0.12 & 0.27 & 0.15 & $\mathrm{~W}$ & 49 \\
\hline 168 & 4237.891 & Ti I & 16 & 4.0 & 0.38 & 0.23 & 0.15 & $\mathrm{~S}$ & \\
\hline 169 & 4246.837 & Sc II & 171 & 40.3 & $\begin{array}{l}0.460 \\
0.470\end{array}$ & $\begin{array}{l}0.225 \\
0.225\end{array}$ & $\begin{array}{l}0.235 \\
0.245\end{array}$ & MS & 50 \\
\hline 170 & 4250.130 & $\mathrm{Fe} \mathrm{I}$ & $342^{*}$ & 79.5 & 0.26 & 0.12 & 0.14 & W & \\
\hline 171 & 4254.346 & $\mathrm{Cr}$ I (V II) & 393 & 92.4 & $\begin{array}{l}0.40 \\
0.270\end{array}$ & $\begin{array}{l}0.16 \\
0.225\end{array}$ & $\begin{array}{l}0.24 \\
0.045\end{array}$ & M1 & \\
\hline 172 & 4268.628 & V I & 23 & 5.6 & 0.45 & 0.22 & 0.23 & $\mathrm{~S}$ & \\
\hline 173 & 4271.774 & $\mathrm{Fe}_{\mathrm{I}}$ & 756 & 177 & 0.24 & 0.09 & 0.15 & W & \\
\hline 174 & 4274.806 & $\mathrm{Cr} \mathrm{I}$ & 196 & 45.8 & $\begin{array}{l}0.59 \\
0.36\end{array}$ & $\begin{array}{l}0.22 \\
0.22\end{array}$ & $\begin{array}{l}0.37 \\
0.14\end{array}$ & M1 & \\
\hline 175 & 4283.014 & $\mathrm{Ca} \mathrm{I}$ & 133 & 31.0 & 0.33 & 0.18 & 0.15 & W & 51 \\
\hline 176 & 4289.922 & Fe I (Ce II) & 65 & 19.1 & 0.285 & 0.170 & 0.115 & S & \\
\hline 177 & 4299.249 & /Fe I Ti I (CH) & 212 & 49.3 & 0.22 & 0.12 & 0.10 & W & 52 \\
\hline 178 & 4307.748 & $\mathrm{Ca} \mathrm{I}$ & 59 & 40.2 & 0.14 & 0.04 & 0.10 & S & 53 \\
\hline 179 & 4307.912 & /Fe I Ti II (CH) & 723 & 165 & 0.14 & 0.04 & 0.10 & $\mathrm{~W}$ & 54 \\
\hline 180 & 4325.775 & $\mathrm{Fe}$ I (Fe I p) & 793 & 174 & $\begin{array}{l}0.32 \\
0.380\end{array}$ & $\begin{array}{l}0.12 \\
0.195\end{array}$ & $\begin{array}{l}0.20 \\
0.185\end{array}$ & M1 & \\
\hline 181 & 4339.722 & Cr I & 69 & 23.8 & 0.27 & $0.15^{*}$ & 0.12 & $\mathrm{~S}$ & \\
\hline 182 & 4340.475 & $\mathrm{H} \mathrm{I}\left[\mathrm{H}_{\gamma}\right]$ & 2855 & 659 & 0.25 & 0.13 & 0.12 & W & \\
\hline 183 & 4354.436 & La II & 13 & 3.4 & 0.295 & $0.195^{*}$ & 0.100 & S & 55 \\
\hline 184 & 4374.944 & Y II & 88 & 20.1 & 0.39 & 0.18 & 0.21 & S & \\
\hline
\end{tabular}

46 At line center this signal shows quite a complex profile with several secondary peaks and dips.

47 This line is severely blended with a weaker Ce II line. In the absence of this blend, the amplitude of $Q / I$ peak might be different.

48 At line center this signal shows quite a complex profile with several secondary peaks and dips.

49 It is not possible to exclude that the "W" shape of this signal could be due to the depolarization produced by several blending lines.

50 The polarization profile shows a complex structure (that might be due to hyperfine structure), and an extended blue wing.

51 The depolarization in the blue wing of this profile is rather peculiar. For this reason the reference level for measuring the signal amplitude has been taken in the dip of the red wing.

52 It is difficult to measure the amplitude of this signal because of the presence of several blends.

53 It is difficult to decide whether this line has to be classified as "S" or "W" because of the blend with the /Fe I Ti I (CH) line at $4307.912 \AA$, and because the polarization signal is quite noisy around this wavelength.

54 At line center this line shows a two-peak structure, probably due to the blend.

55 The polarization peak does not fall exactly at line center, probably because of the presence of a strong blend with a Mg I line. It is not possible to exclude that in the absence of this blend, the $Q / I$ peak amplitude would have been significantly larger. 
Table 1. continued.

\begin{tabular}{|c|c|c|c|c|c|c|c|c|c|}
\hline \# & $\begin{array}{l}\lambda^{\text {Sun }} \\
(\AA)\end{array}$ & Elem. & $\begin{array}{c}\mathrm{W}_{\lambda} \\
(\mathrm{m} \AA)\end{array}$ & $\begin{array}{c}\mathrm{W}_{\lambda} / \lambda \\
(\mathrm{F})\end{array}$ & $\begin{array}{c}(Q / I)^{\mathrm{M}} \\
(\%)\end{array}$ & $\begin{array}{c}(Q / I)^{\mathrm{m}} \\
(\%)\end{array}$ & $\begin{array}{l}Q / I \\
(\%)\end{array}$ & Type & Notes \\
\hline 185 & 4383.557 & $\mathrm{Fe} \mathrm{I}$ & 1008 & 235 & $\begin{array}{l}0.410 \\
0.305\end{array}$ & $\begin{array}{l}0.110 \\
0.175\end{array}$ & $\begin{array}{l}0.300 \\
0.130\end{array}$ & M1 & 56 \\
\hline 186 & 4404.761 & $\mathrm{Fe} \mathrm{I}$ & 898 & 181 & $\begin{array}{l}0.29 \\
0.23\end{array}$ & $\begin{array}{l}0.06 \\
0.17\end{array}$ & $\begin{array}{l}0.23 \\
0.06\end{array}$ & M1 & \\
\hline 187 & 4415.135 & $\mathrm{Fe} \mathrm{I}$ & $417^{*}$ & 92.9 & 0.07 & 0.20 & 0.13 & W & \\
\hline 188 & 4425.444 & $\mathrm{Ca} \mathrm{I}$ & 145 & 31.6 & 0.275 & 0.165 & 0.110 & $\mathrm{~S}$ & \\
\hline 189 & 4431.292 & Ti I & 5 & 1.1 & 0.38 & 0.16 & 0.22 & S & 57 \\
\hline 190 & 4453.710 & Ti I & 36 & 9.0 & 0.280 & 0.155 & 0.125 & $S$ & \\
\hline 191 & 4460.225 & Ce II & $100^{*}$ & 6.0 & 0.315 & 0.155 & 0.160 & S & 58 \\
\hline 192 & 4462.993 & $\mathrm{Nd}$ II & 13 & 2.9 & 0.26 & 0.15 & 0.11 & $S$ & \\
\hline 193 & 4471.244 & Ti I (Ce II) & 35 & 8.5 & 0.265 & $0.165^{*}$ & 0.100 & $S$ & \\
\hline 194 & 4486.914 & Ce II & 11 & 2.5 & 0.28 & 0.15 & 0.13 & S & \\
\hline 195 & 4500.288 & Cr I & $39^{*}$ & 6.2 & 0.230 & 0.145 & 0.085 & S & \\
\hline 196 & 4511.900 & $\mathrm{Cr} \mathrm{I}$ & 31 & 8.4 & 0.180 & $0.125^{*}$ & 0.055 & $S$ & \\
\hline 197 & 4527.325 & $/ / \mathrm{Ti}$ I $/ \mathrm{Cr}$ I (Ce II) & 67 & 14.8 & 0.210 & 0.140 & 0.070 & $S$ & \\
\hline 198 & 4536.054 & Ti I & $147^{*}$ & 16.3 & 0.240 & $0.125^{*}$ & 0.115 & $S$ & \\
\hline 199 & 4540.710 & Cr I & 52 & 12.3 & 0.165 & $0.110^{*}$ & 0.055 & S & \\
\hline 200 & 4554.036 & Ba II & 159 & 36.7 & $\begin{array}{l}0.78 \\
0.50\end{array}$ & $\begin{array}{l}0.13 \\
0.13\end{array}$ & $\begin{array}{l}0.65 \\
0.37\end{array}$ & MS & \\
\hline 201 & 4572.284 & Ce II & 15 & 3.3 & 0.205 & 0.130 & 0.075 & S & \\
\hline 202 & 4595.593 & $\mathrm{Cr} \mathrm{I}$ & 21 & 4.6 & 0.255 & $0.105^{*}$ & 0.150 & $S$ & \\
\hline 203 & 4599.227 & Ti I & 6 & 1.3 & 0.185 & 0.120 & 0.065 & $S$ & \\
\hline \multirow[t]{2}{*}{204} & 4607.338 & Sr I & 36 & 7.8 & 1.200 & 0.120 & 1.080 & $\mathrm{~S}$ & \\
\hline & & & \multicolumn{2}{|c|}{ - Volume I } & & & & & 59 \\
\hline 205 & 4639.368 & Ti I & 36 & 8.4 & 0.185 & 0.115 & 0.070 & $S$ & \\
\hline 206 & 4639.946 & Ti I & 31 & 7.3 & 0.175 & 0.115 & 0.060 & $\mathrm{~S}$ & \\
\hline 207 & 4679.983 & $\mathrm{Fe} I \mathrm{p}$ & 6.5 & 1.4 & 0.155 & 0.105 & 0.050 & S & \\
\hline 208 & 4703.003 & Mg I & 326 & 71.9 & 0.095 & 0.045 & 0.050 & $\mathrm{~W}$ & \\
\hline 209 & 4742.798 & Ti I & 27 & 5.7 & 0.290 & 0.098 & 0.192 & $\mathrm{~S}$ & \\
\hline 210 & 4758.124 & Ti I & 40 & 8.2 & 0.202 & 0.095 & 0.107 & S & \\
\hline 211 & 4759.276 & Ti I & 41 & 8.6 & 0.192 & 0.095 & 0.097 & S & \\
\hline 212 & 4778.258 & Ti I & 16 & 3.3 & 0.148 & 0.092 & 0.056 & S & \\
\hline 213 & 4796.189 & $\mathrm{Cr} \mathrm{I}-/ / \mathrm{Ti} \mathrm{I}$ & 24 & 5.0 & 0.145 & 0.090 & 0.055 & $S$ & \\
\hline 214 & 4799.794 & V I-//Ti I & $75^{*}$ & 10.2 & 0.175 & 0.089 & 0.086 & S & \\
\hline 215 & 4805.420 & Ti I & 37 & 7.7 & 0.150 & 0.087 & 0.063 & S & \\
\hline 216 & 4840.884 & Ti I & 60 & 12.6 & 0.136 & 0.082 & 0.054 & $S$ & 60 \\
\hline 217 & 4868.263 & Ti I & 26 & 5.5 & 0.130 & 0.080 & 0.050 & $\mathrm{~S}$ & \\
\hline 218 & 4975.351 & Ti I & $35^{*}$ & 3.6 & 0.165 & 0.070 & 0.095 & $S$ & 61 \\
\hline 219 & 5021.923 & $\mathrm{Cr}$ I Fe I C2 & 22 & 4.4 & 0.115 & 0.065 & 0.050 & $S$ & \\
\hline 220 & 5025.764 & $\mathrm{Fe}$ I C2 & 11 & 2.2 & 0.124 & 0.063 & 0.061 & S & \\
\hline 221 & 5112.279 & $\mathrm{Zr}$ II & 8 & 1.6 & 0.106 & 0.056 & 0.050 & S & \\
\hline
\end{tabular}

56 At line center this line shows a complex structure, with several secondary peaks and dips.

57 This line is severely blended with a Sc II line. In the absence of this blend the amplitude of the $Q / I$ signal might be larger.

58 This line is severely blended with a V I line. In the absence of this blend the amplitude of the $Q / I$ signal might be larger.

59 Volume II of the atlas covers the spectral interval between $3910 \AA$ and $4630 \AA$, Vol. I the spectral interval between $4625 \AA$ and $6995 \AA$. The spectral interval between $4624 \AA$ and $4626.5 \AA$ is covered by both volumes. Comparing the observations made in this spectral interval, it can be noticed that the depolarization produced by two rather intense lines (Fe I and Cr I) is larger in Vol. I than in Vol. II. As already discussed in the note 41 , this might be due to the fact that, although the nominal position of the slit is $\mu=0.1$ for the observations of both atlases, because of the seeing the "effective" position might be different from the nominal value.

${ }^{60}$ A secondary peak is observed to the blue of line center. The observation in this spectral region is quite noisy, and at this level it is not possible to exclude that the signal could be of "W" type.

${ }^{61}$ This line is severely blended with a Fe I line. In the absence of this blend the amplitude of the $Q / I$ signal might be larger. 
Table 1. continued.

\begin{tabular}{|c|c|c|c|c|c|c|c|c|c|}
\hline$\#$ & $\begin{array}{l}\lambda^{\text {Sun }} \\
(\AA)\end{array}$ & Elem. & $\begin{array}{c}\mathrm{W}_{\lambda} \\
(\mathrm{m} \AA)\end{array}$ & $\begin{array}{c}\mathrm{W}_{\lambda} / \lambda \\
(\mathrm{F})\end{array}$ & $\begin{array}{c}(Q / I)^{\mathrm{M}} \\
(\%)\end{array}$ & $\begin{array}{c}(Q / I)^{\mathrm{m}} \\
(\%)\end{array}$ & $\begin{array}{l}Q / I \\
(\%)\end{array}$ & Type & Notes \\
\hline 222 & 5113.127 & $/ \mathrm{Cr}$ I MgH & 23 & 4.5 & 0.120 & 0.056 & 0.064 & $S$ & \\
\hline 223 & 5115.790 & $\mathrm{Fe} I$ & 23 & 4.5 & 0.120 & 0.056 & 0.064 & S & 62 \\
\hline 224 & 5120.420 & Ti I & 31 & 6.0 & 0.135 & 0.055 & 0.080 & S & 63 \\
\hline 225 & 5138.717 & $\mathrm{Cr} \mathrm{I}-\mathrm{MgH}$ & 13 & 2.4 & 0.130 & 0.055 & 0.075 & S & \\
\hline 226 & 5139.648 & Cr I & 46 & 8.9 & 0.120 & 0.054 & 0.066 & S & 64 \\
\hline 227 & 5146.119 & $\mathrm{Fe}$ II $\mathrm{p} \mathrm{C} 2$ & 37 & 6.6 & 0.140 & 0.054 & 0.086 & S & 65 \\
\hline 228 & 5149.796 & Co I MgH & 11 & 2.1 & 0.126 & 0.053 & 0.073 & S & \\
\hline 229 & 5153.241 & $\mathrm{Cu} \mathrm{I}$ & $56^{*}$ & 8.5 & 0.163 & 0.053 & 0.110 & S & 66 \\
\hline 230 & 5167.327 & Mg I & $935^{*}$ & 173 & 0.130 & 0.030 & 0.100 & W & \\
\hline 231 & 5171.028 & $\mathrm{Ru}$ I MgH & 5 & 1.0 & 0.110 & 0.052 & 0.058 & S & \\
\hline 232 & 5176.792 & /V I-MgH & 10 & 1.9 & 0.115 & 0.051 & 0.064 & S & \\
\hline 233 & 5196.452 & Cr I & 34 & 6.5 & 0.090 & $0.040^{*}$ & 0.050 & S & 67 \\
\hline 234 & 5206.044 & Cr I (Ti I) & $216^{*}$ & 36.6 & $\begin{array}{l}0.115 \\
0.115\end{array}$ & $\begin{array}{l}0.050 \\
0.050\end{array}$ & $\begin{array}{l}0.065 \\
0.065\end{array}$ & MS & 68 \\
\hline 235 & 5208.432 & $\mathrm{Cr} \mathrm{I}$ & 247 & 47.4 & $\begin{array}{l}0.110 \\
0.077\end{array}$ & $\begin{array}{l}0.040 \\
0.050\end{array}$ & $\begin{array}{l}0.070 \\
0.027\end{array}$ & MS & 68 \\
\hline 236 & 5218.209 & $\mathrm{Cu} \mathrm{I}$ & 48 & 9.8 & 0.101 & 0.049 & 0.052 & S & \\
\hline 237 & 5239.823 & Sc II & 55 & 10.5 & 0.117 & 0.048 & 0.069 & S & \\
\hline 238 & 5387.565 & $\mathrm{Cr} \mathrm{I}$ & $25^{*}$ & 1.8 & 0.100 & 0.040 & 0.060 & S & 69 \\
\hline 239 & 5528.418 & Mg I & 293 & 53.8 & 0.052 & 0.000 & 0.052 & W & \\
\hline 240 & 5535.51 & $\mathrm{Ba} \mathrm{I}$ & $113^{*}$ & & 0.165 & 0.032 & 0.133 & $\mathrm{~S}$ & \\
\hline 241 & 5565.485 & Ti I & 16 & 2.9 & 0.150 & 0.031 & 0.119 & S & \\
\hline 242 & 5598.491 & $\mathrm{Ca} \mathrm{I}$ & 118 & 21.1 & 0.080 & 0.030 & 0.050 & S & \\
\hline 243 & 5644.146 & Ti I & 29 & 5.1 & 0.260 & 0.029 & 0.231 & S & \\
\hline 244 & 5889.973 & $\mathrm{Na} \mathrm{I}\left[\mathrm{D}_{2}\right]$ & $752^{*}$ & 120 & $\begin{array}{l}0.330 \\
0.220\end{array}$ & $\begin{array}{l}0.021 \\
0.021\end{array}$ & $\begin{array}{l}0.309 \\
0.199\end{array}$ & MS & \\
\hline 245 & 5895.940 & $\mathrm{Na} \mathbf{I}\left[\mathrm{D}_{1}\right]$ & $564^{*}$ & 91.0 & 0.05 & -0.02 & 0.07 & - & 70 \\
\hline 246 & 6024.068 & $\mathrm{Fe} \mathrm{I}$ & $117^{*}$ & 19.8 & 0.060 & $0.010^{*}$ & 0.050 & $S$ & \\
\hline 247 & 6141.727 & $/ \mathrm{Ba}$ II-Fe I & 113 & 19.4 & 0.059 & 0.000 & 0.059 & W & \\
\hline 248 & 6562.808 & $\mathrm{H} \mathrm{I}\left[\mathrm{H}_{\alpha}\right]$ & 4020 & 649 & 0.120 & 0.010 & 0.110 & $\mathrm{~S}$ & 71 \\
\hline
\end{tabular}

62 This line has an extremely weak Einstein coefficient $\left(5.15 \times 10^{4} \mathrm{~s}^{-1}\right)$, and it is not possible to exclude that this polarization signal might be due to a $\mathrm{C}_{2}$ line.

63 The polarization peak does not fall exactly at line center, probably because of the presence of a strong blend with a Fe II $\mathrm{p} \mathrm{C}_{2}$ line. It is not possible to exclude that in the absence of this blend, the $Q / I$ peak amplitude would have been larger.

64 This line is severely blended with a Fe I line. In the absence of this blend the amplitude of the $Q / I$ signal might be larger.

65 This polarization signal is quite wide. This may be due to the presence of a blending $\mathrm{C}_{2}$ line.

${ }^{66}$ The large width of this polarization profile, and the fact that the peak does not fall at line center, suggest that the blending $\mathrm{C}_{2}$ line might affect significantly this signal.

67 The signal is somewhat noisy in this spectral region, and it is not possible to exclude that the signal might be classified as "W".

68 Very likely the overall structure of the polarization signal in the interval between $5204 \AA$ and $5210 \AA$ is due to quantum interferences between the $J$-levels of the term ${ }^{5} \mathrm{P}^{\mathrm{o}}$. Both lines seem to show an "MS" structure partially hidden by blends.

69 This line is severely blended with a Fe I line. In the absence of this blend the amplitude of the $Q / I$ signal might be larger.

70 This signal cannot be classified according to the scheme proposed in this work.

71 According to our scheme, this polarization signal has to be classified as "S". However, it has to be remarked that its width is much larger than the width of any other signal of this class. 
L. Belluzzi and E. Landi Degl'Innocenti: Analysis of the most polarizing lines of the 2nd solar spectrum, Online Material p 9

焉

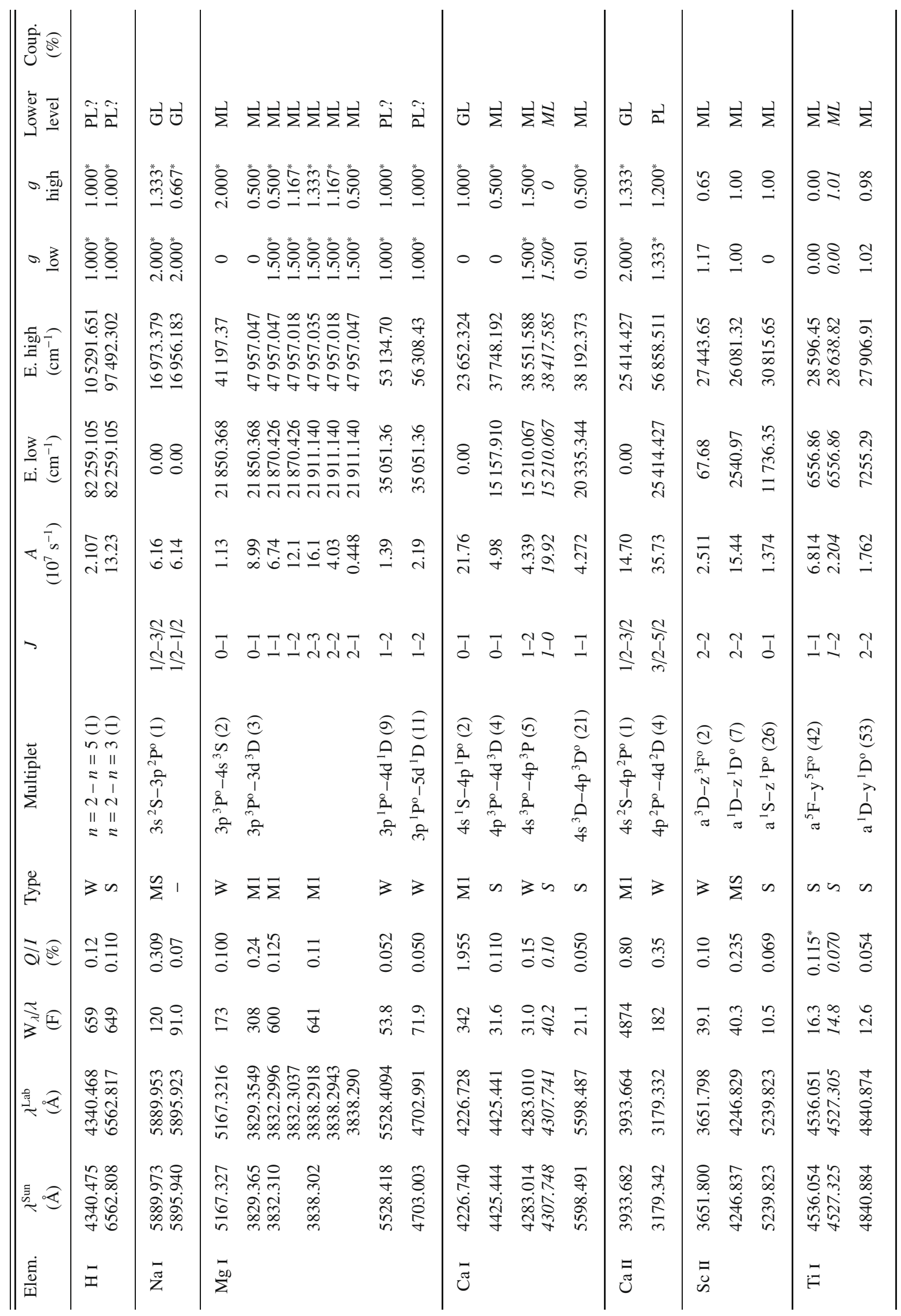

突 


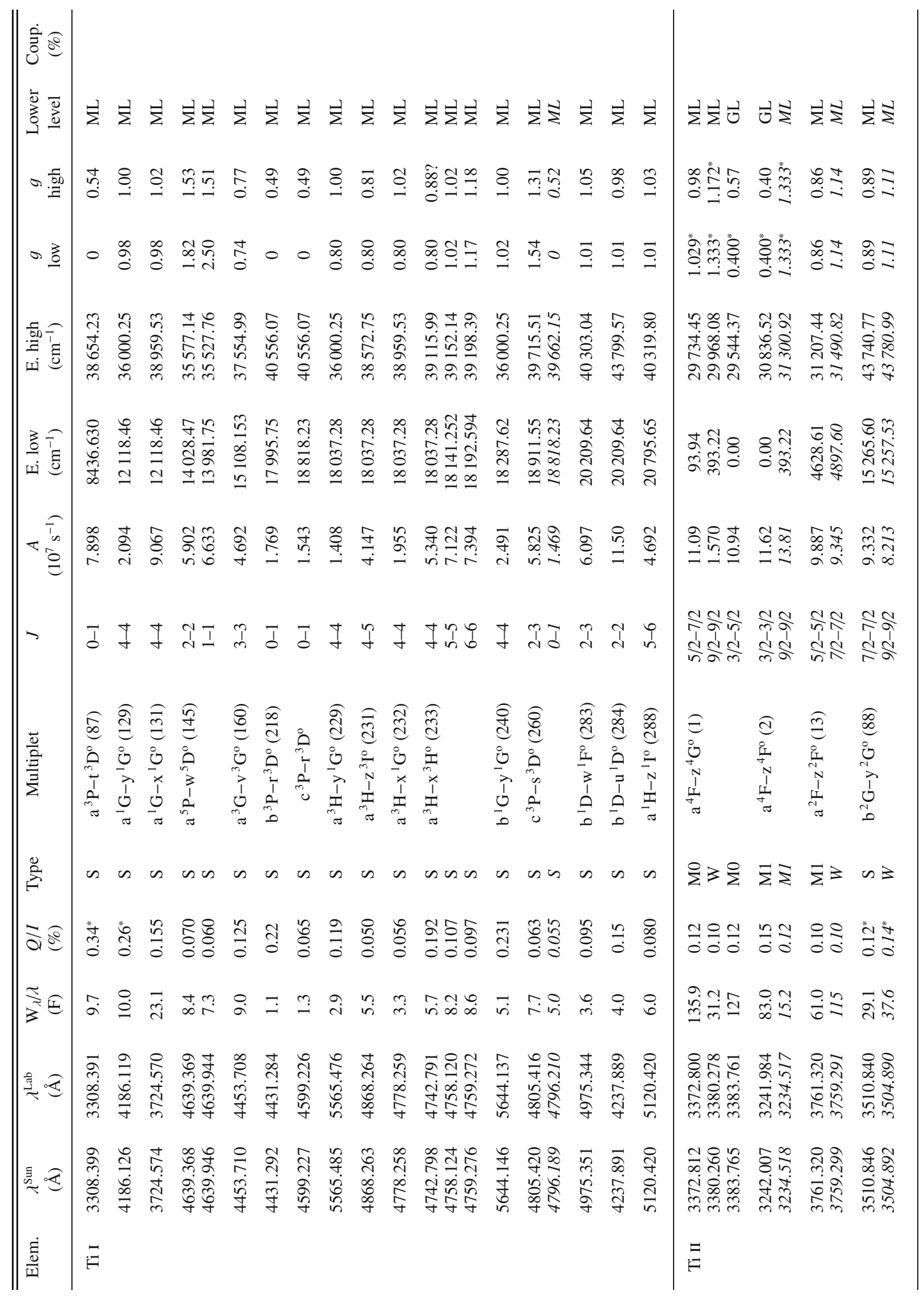




\begin{tabular}{|c|c|c|c|c|c|c|c|c|c|c|c|c|c|c|c|}
\hline 递 & $\xi$ & $\xi$ & ્ 어 & ت઼ & $\dot{z}$ & $\xi$ & $\dot{\Sigma}$ & $\dot{\Sigma} \bar{\Sigma} \dot{z}$ & $\xi$ & $\sum \xi$ & $\vec{\Sigma}$ & $\sum$ & تี ت઼ & $\sum \xi \xi$ & 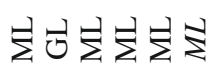 \\
\hline $0 . \frac{50}{3}$ & $\stackrel{\text { กิ }}{-}$ & $\stackrel{\infty}{-}$ & ลू & 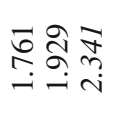 & 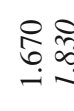 & ठ̊. & ஜ̊ & 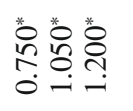 & 苋 & 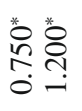 & 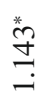 & 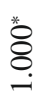 & 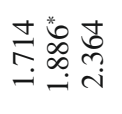 & 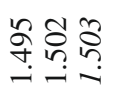 & 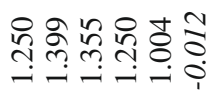 \\
\hline$\infty \frac{3}{0}$ & $\stackrel{\infty}{=}$ & $\stackrel{t}{0}$ & 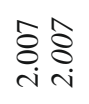 & $\begin{array}{l}\hat{8} \tilde{\delta} \\
i \\
i\end{array}$ & \& & ○ & $\circ$ & 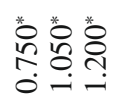 & $\circ$ & 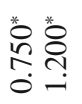 & $\stackrel{\text { go }}{g}$ & 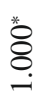 & बे बे बे & 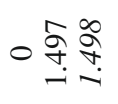 & 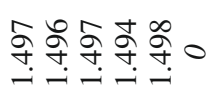 \\
\hline 点 & 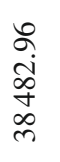 & 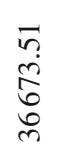 & 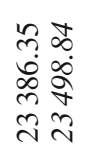 & 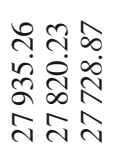 & 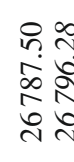 & $\begin{array}{l}0 \\
\substack{\infty \\
\infty \\
\infty \\
\infty}\end{array}$ & $\begin{array}{l}\infty \\
\infty \\
\infty \\
\infty \\
0 \\
i n\end{array}$ & 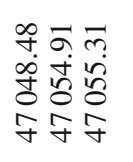 & 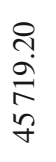 & 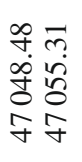 & $\begin{array}{l}0 \\
2 \\
2 \\
i n \\
i n\end{array}$ & $\begin{array}{l}\dot{a} \\
\dot{0} \\
n \\
n \\
i n\end{array}$ & 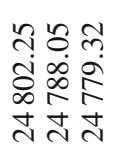 & 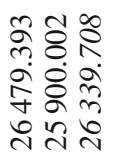 & 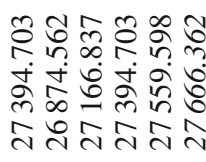 \\
\hline 四 & 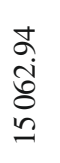 & $\underset{\substack{\hat{q} \\
\infty}}{\hat{\Phi}}$ & :8 & :8: & 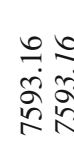 & $\begin{array}{l}\infty \\
\stackrel{\infty}{\circ} \\
\stackrel{\circ}{\kappa}\end{array}$ & 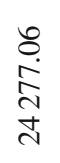 & 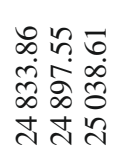 & 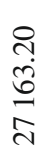 & 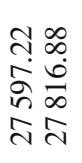 & $\begin{array}{l}8 \\
\infty \\
0 \\
0 \\
m\end{array}$ & 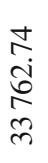 & : $8: 0$ & 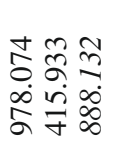 & 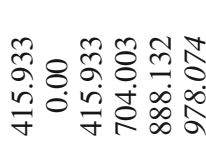 \\
\hline & 包 & $\stackrel{\infty}{\stackrel{+}{+}}$ & 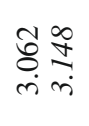 & 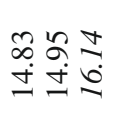 & तु & $\stackrel{+}{\stackrel{+}{0}} \stackrel{0}{0}$ & $\begin{array}{l}0 \\
\stackrel{0}{a} \\
\text { i }\end{array}$ & 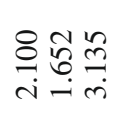 & 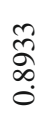 & 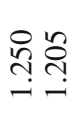 & : & 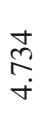 & 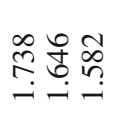 & 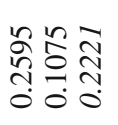 & 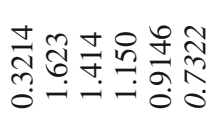 \\
\hline- & $\begin{array}{l}\frac{9}{9} \\
\frac{9}{9} \\
\frac{9}{4}\end{array}$ & $\hat{n}$ & $m=$ & $\begin{array}{l}\tan \\
\text { लिक }\end{array}$ & in & $\bar{d}$ & $\bar{d}$ & nftin & $\vec{\jmath}$ & $\begin{array}{ll}m & n \\
m & i n\end{array}$ & I & $\begin{array}{l}0 \\
l\end{array}$ & 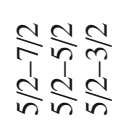 & JII & mita \\
\hline$\frac{\overrightarrow{0}}{\frac{\overrightarrow{0}}{2}}$ & 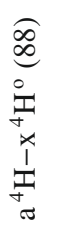 & 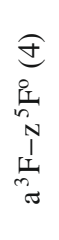 & $\begin{array}{l}0 \\
0 \\
\vdots \\
N \\
i \\
n \\
0\end{array}$ & 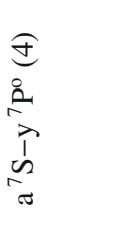 & 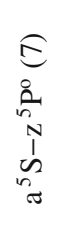 & $\begin{array}{l}\text { త్ } \\
\text { in } \\
\text { in } \\
\text { I } \\
\text { in } \\
\text { in }\end{array}$ & $\begin{array}{l}\underset{\infty}{\infty} \\
\stackrel{D}{=} \\
\stackrel{D}{D} \\
n \\
1 \\
1 \\
0 \\
0\end{array}$ & 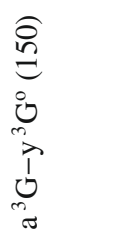 & 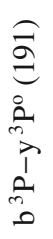 & 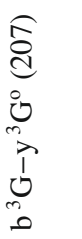 & 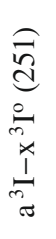 & 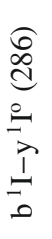 & 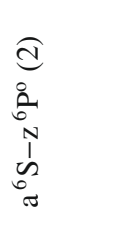 & 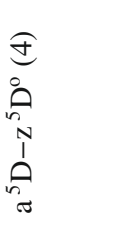 & 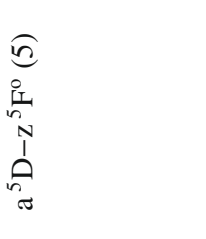 \\
\hline 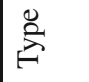 & $n$ & 3 & $\sum \Sigma$ & $\bar{\Sigma} \equiv$ & $\sum_{1}^{n}=$ & is & is & isen & $n$ & $n \infty$ & $n$ & is & 333 & 333 & $3 \sum^{2} 333 n$ \\
\hline $\bar{a} @$ & กู่ & $\stackrel{\circ}{\circ}$ & 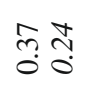 & 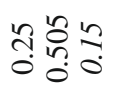 & ㅁำ & $\stackrel{*}{\dddot{0}}$ & $\stackrel{*}{\tilde{n}}$ & $\begin{array}{l}n \\
0 \\
0 \\
0 \\
0 \\
0 \\
0\end{array}$ & $\stackrel{8}{0}$ & 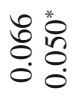 & $\begin{array}{l}\frac{8}{0} \\
0\end{array}$ & 草 & $\hat{\imath}=0$ & חָ & 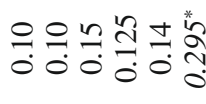 \\
\hline$\stackrel{5}{3}$ & $\begin{array}{l}\dot{0} \\
i\end{array}$ & $\stackrel{\circ}{\sim}$ & $\begin{array}{l}\infty \\
\dot{q} \\
\dot{q} \\
\dot{\alpha}\end{array}$ & $\stackrel{\Im}{\beth} \cong$ & 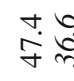 & $\stackrel{\infty}{\infty}$ & 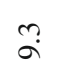 & 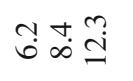 & $\stackrel{\infty}{-}$ & के & $\stackrel{+}{\circ}$ & $\stackrel{\circ}{+}$ & 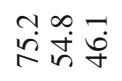 & 宣吉过 & 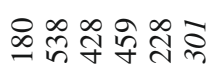 \\
\hline & 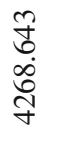 & 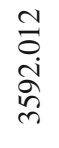 & 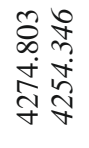 & 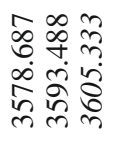 & 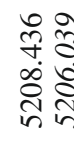 & $\stackrel{\infty}{\stackrel{\infty}{\stackrel{े}{े}}}$ & $\begin{array}{l}0 \\
\stackrel{0}{0} \\
\infty \\
\infty \\
\infty \\
\infty\end{array}$ & 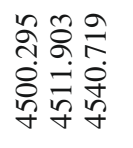 & $\begin{array}{l}n \\
\hat{n} \\
\infty \\
\infty \\
n\end{array}$ & 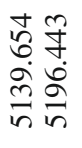 & $\frac{8}{\circ}$ & 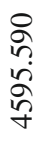 & 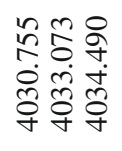 & 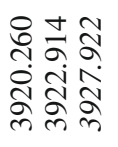 & 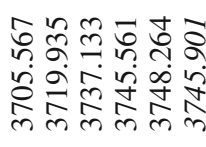 \\
\hline & 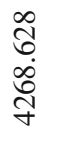 & $\begin{array}{l}\hat{\delta} \\
\text { ¿े } \\
\text { ळे }\end{array}$ & 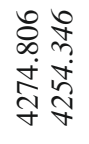 & 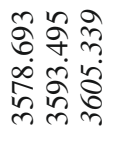 & 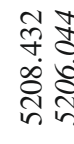 & $\begin{array}{l}\text { ה̃ } \\
\text { ले } \\
\text { ণे }\end{array}$ & $\begin{array}{l}\hat{\sigma} \\
\stackrel{0}{0} \\
\infty \\
\infty \\
\infty\end{array}$ & 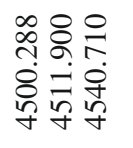 & 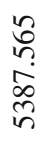 & 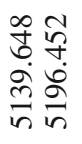 & $\begin{array}{l}\stackrel{0}{\circ} \\
\stackrel{0}{0} \\
\text { के }\end{array}$ & 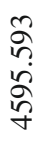 & 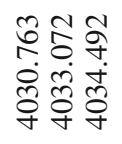 & 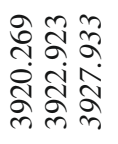 & 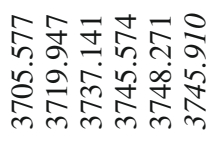 \\
\hline$\frac{\dot{\vec{\theta}}}{\frac{\vec{\omega}}{I}}$ & 5 & 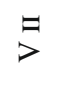 & ت̃ & & & & & & & & & & $\bar{\Sigma}$ & $\overline{0}$ & \\
\hline
\end{tabular}


L. Belluzzi and E. Landi Degl'Innocenti: Analysis of the most polarizing lines of the 2nd solar spectrum, Online Material p 12

\begin{tabular}{|c|c|c|c|c|c|c|c|c|c|c|}
\hline 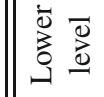 & $\sum \xi$ & $\dot{\Sigma} \vec{\Sigma} \dot{\Sigma} \dot{\Sigma} \vec{\Sigma}$ & 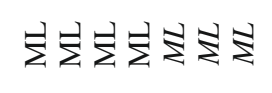 & 로롤 & 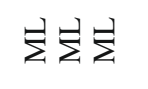 & 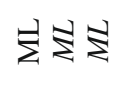 & 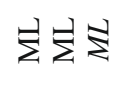 & 로롤 & $\dot{z}$ & $\vec{\Sigma}$ \\
\hline 0.50 & 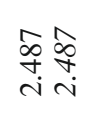 & 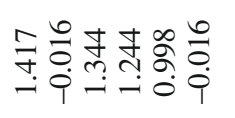 & 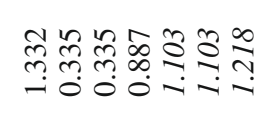 & \& & 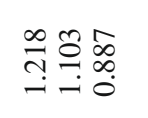 & 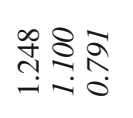 & 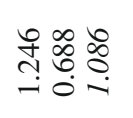 & 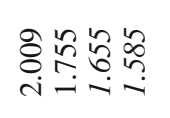 & $\stackrel{n}{a}$ & $\underset{n}{i n n}$ \\
\hline 0 음 & $\circ \stackrel{\infty}{\stackrel{\infty}{+}}$ & 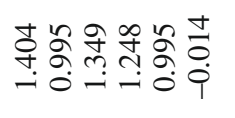 & 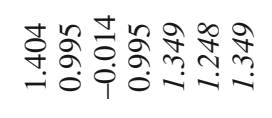 & 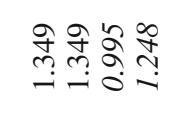 & 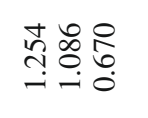 & 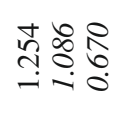 & 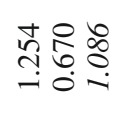 & 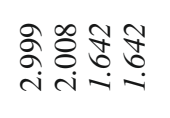 & $\underset{\ddot{c}}{\vec{g}}$ & 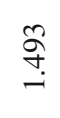 \\
\hline 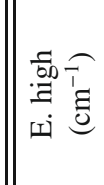 & 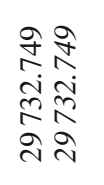 & 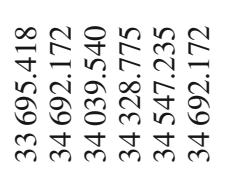 & 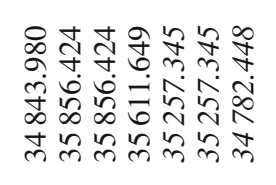 & 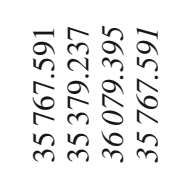 & 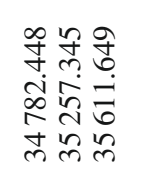 & 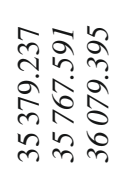 & 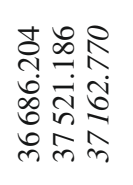 & 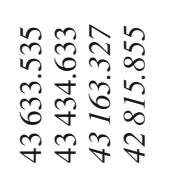 & 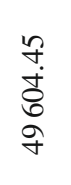 & $\begin{array}{l}n \\
\tilde{o} \\
\infty \\
\infty \\
\infty \\
0 \\
i\end{array}$ \\
\hline 春 & 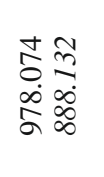 & 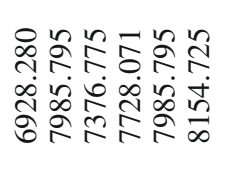 & 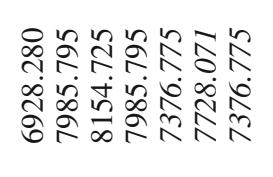 & 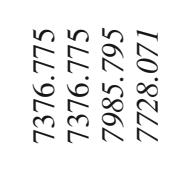 & 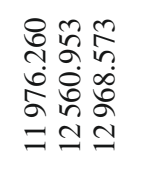 & 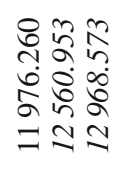 & 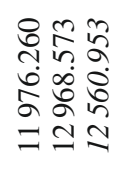 & 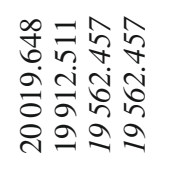 & $\begin{array}{l}\hat{\sigma} \\
\bar{a} \\
\sigma \\
\vec{\alpha}\end{array}$ & 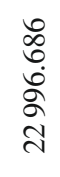 \\
\hline 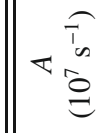 & 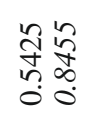 & 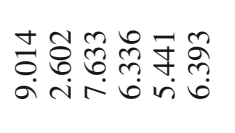 & $\begin{array}{l}\infty \\
0 \\
0 \\
0\end{array}$ & 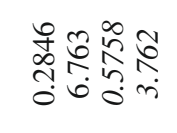 & 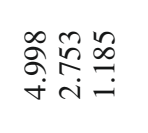 & 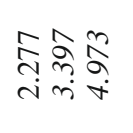 & 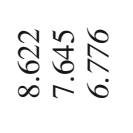 & 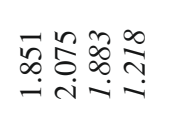 & $\begin{array}{l}\hat{o} \\
\dot{n}\end{array}$ & $\underset{i}{\stackrel{t}{i}}$ \\
\hline- & II & ñ寸 & 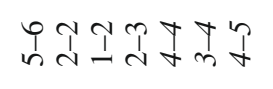 & $\begin{array}{l}\ln \\
\tan 4\end{array}$ & भूत & ind & Iतn & Ind & in & I \\
\hline 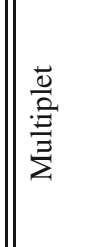 & 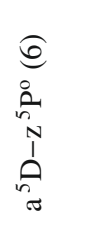 & 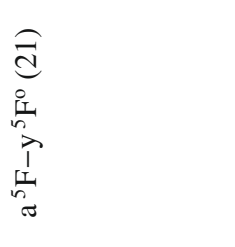 & 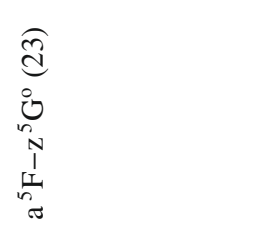 & 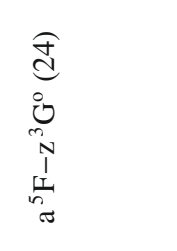 & 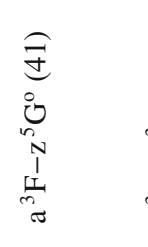 & 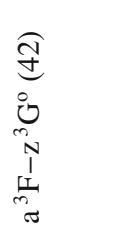 & 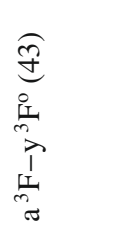 & 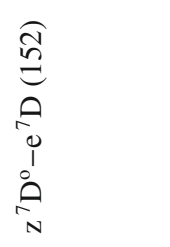 & 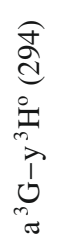 & 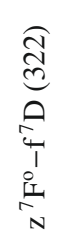 \\
\hline$\stackrel{0}{\stackrel{2}{*}}$ & 33 & $3 B z \bar{\Sigma} \bar{\Sigma} \bar{\Sigma}$ & $\bar{\Sigma} \geqslant \bar{\Sigma} \bar{\Sigma} \Xi z$ & $B \bar{\Sigma} 33$ & $\bar{\Sigma} \bar{\Sigma} z$ & $3 \geq z$ & $\bar{\Sigma} \bar{\Sigma}$ & 3313 & 3 & 3 \\
\hline $\bar{a} @$ & $\frac{n}{0}=$ & 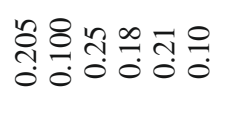 & 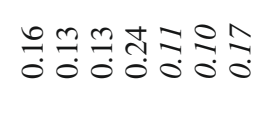 & $\frac{m}{0} \div \frac{\infty}{0}: \frac{1}{0}$ & 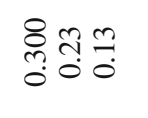 & 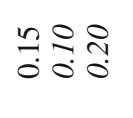 & 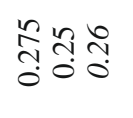 & $\frac{0}{0}: \frac{2}{0}: \frac{0}{0}$ & $\stackrel{\circ}{0}$ & $\frac{m}{0}$ \\
\hline$\underset{3}{\frac{5}{3}}$ & $\stackrel{\infty}{2} \stackrel{\infty}{\sim}$ & 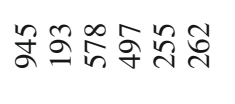 & 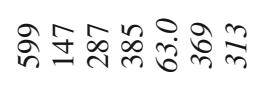 & 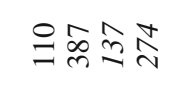 & ๗ูગ & ミ气さ & 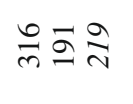 & 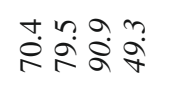 & $\stackrel{0}{\dot{q}}$ & $\stackrel{\infty}{\dot{q}}$ \\
\hline$\stackrel{9}{\sim}$ & 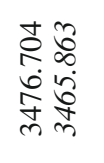 & 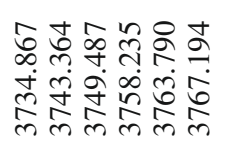 & 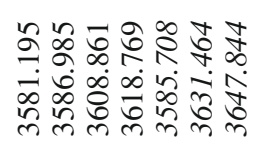 & 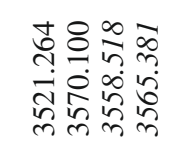 & 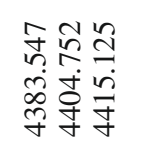 & 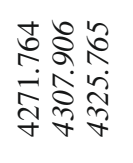 & 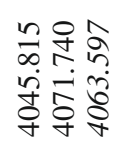 & 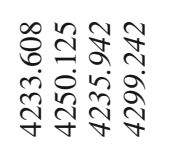 & 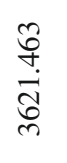 & $\begin{array}{l}\tilde{\sigma} \\
\tilde{b} \\
\dot{\sigma} \\
\tilde{n}\end{array}$ \\
\hline 言这 & 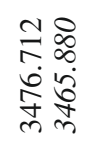 & 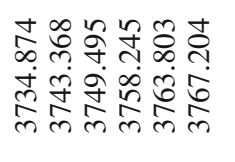 & 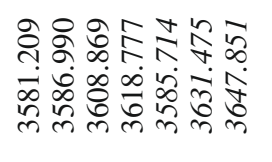 & 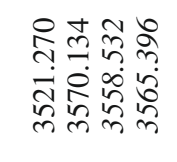 & 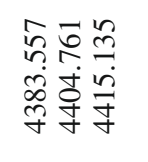 & 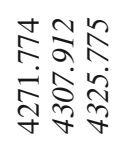 & 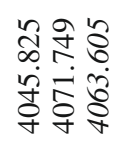 & 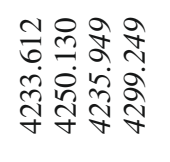 & & $\begin{array}{l}\infty \\
\tilde{\sigma} \\
\dot{+} \\
\tilde{n} \\
\tilde{n}\end{array}$ \\
\hline$\frac{\dot{\vec{d}}}{\Gamma}$ & 这 & & & & & & & & & \\
\hline
\end{tabular}




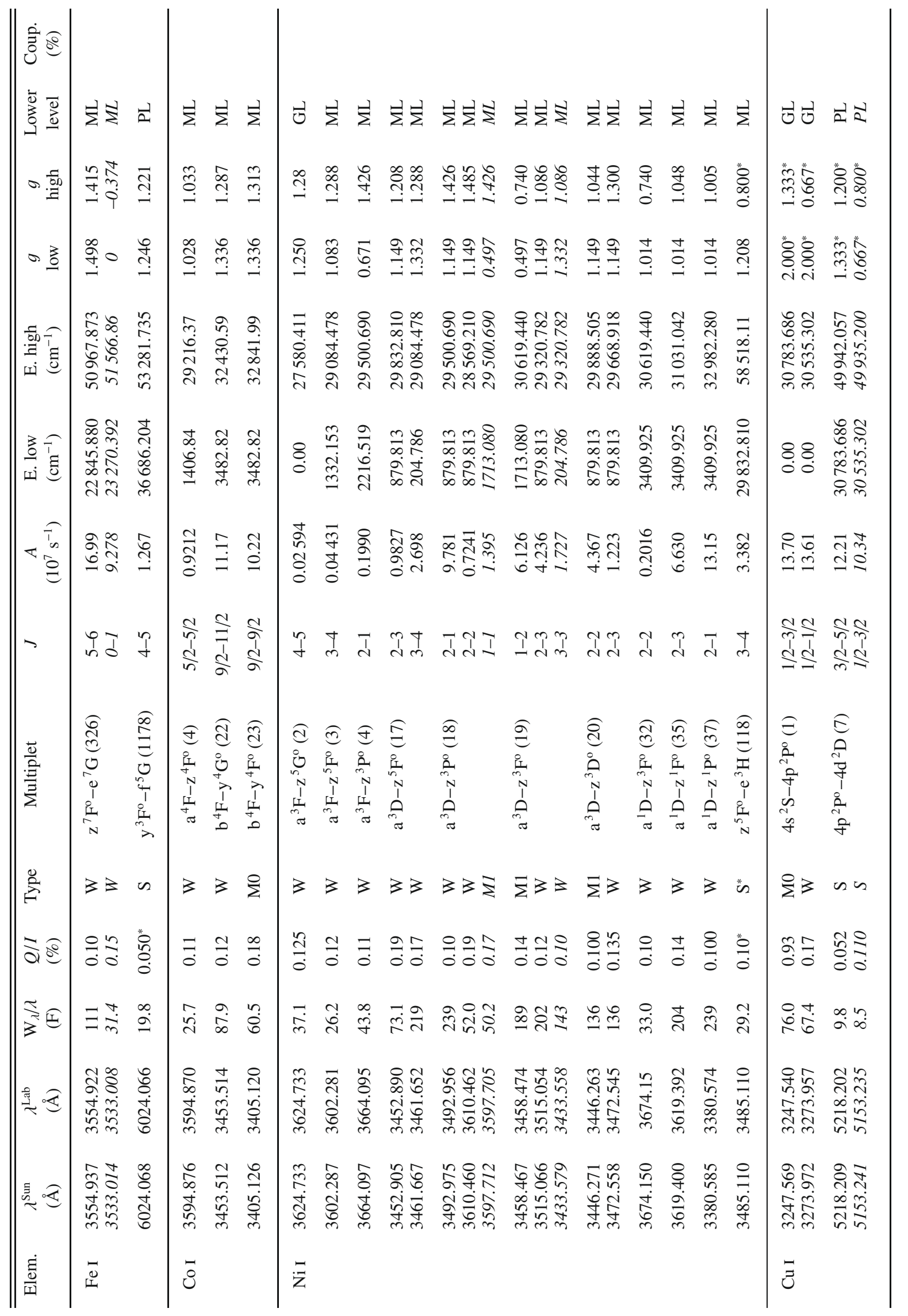




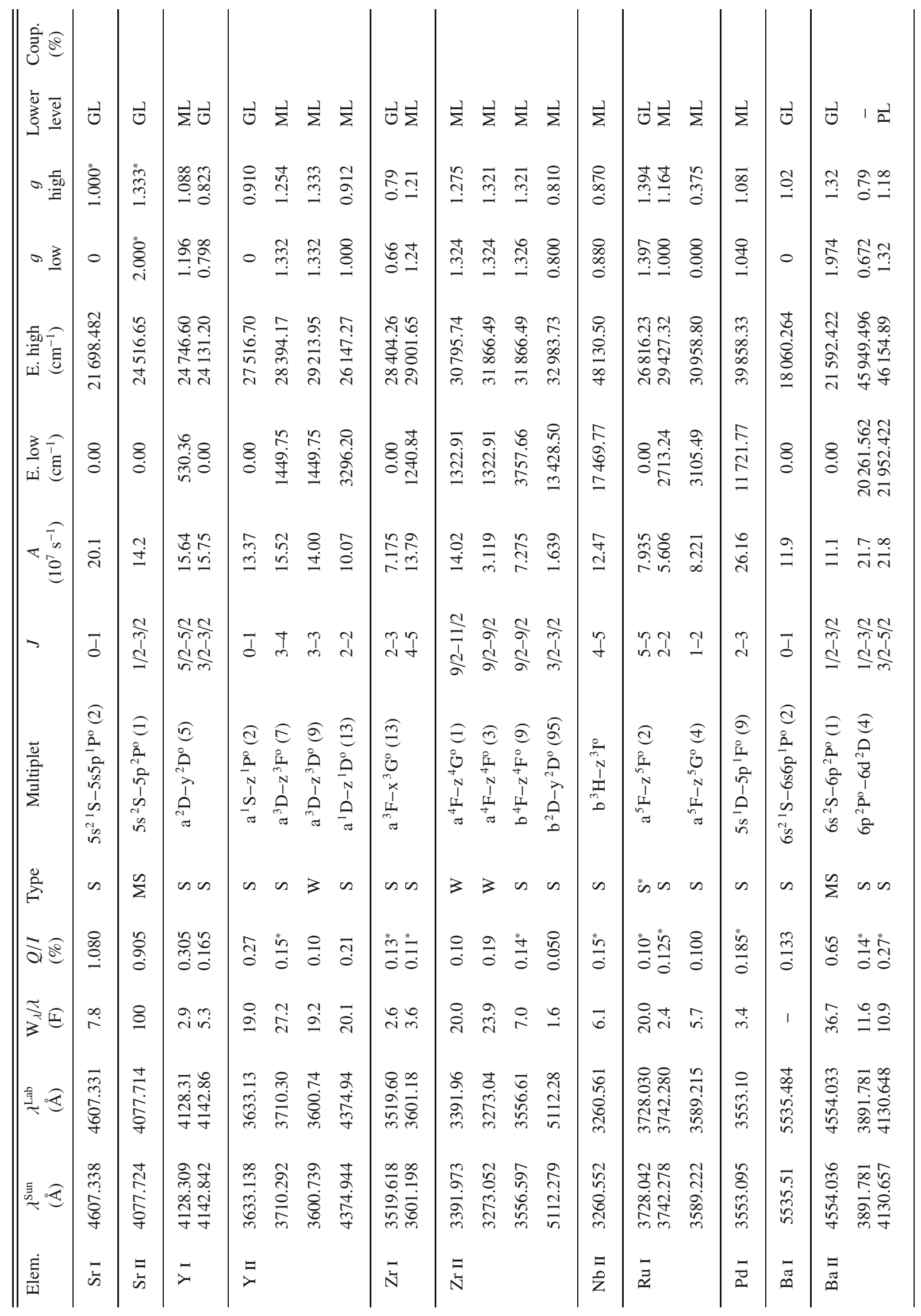




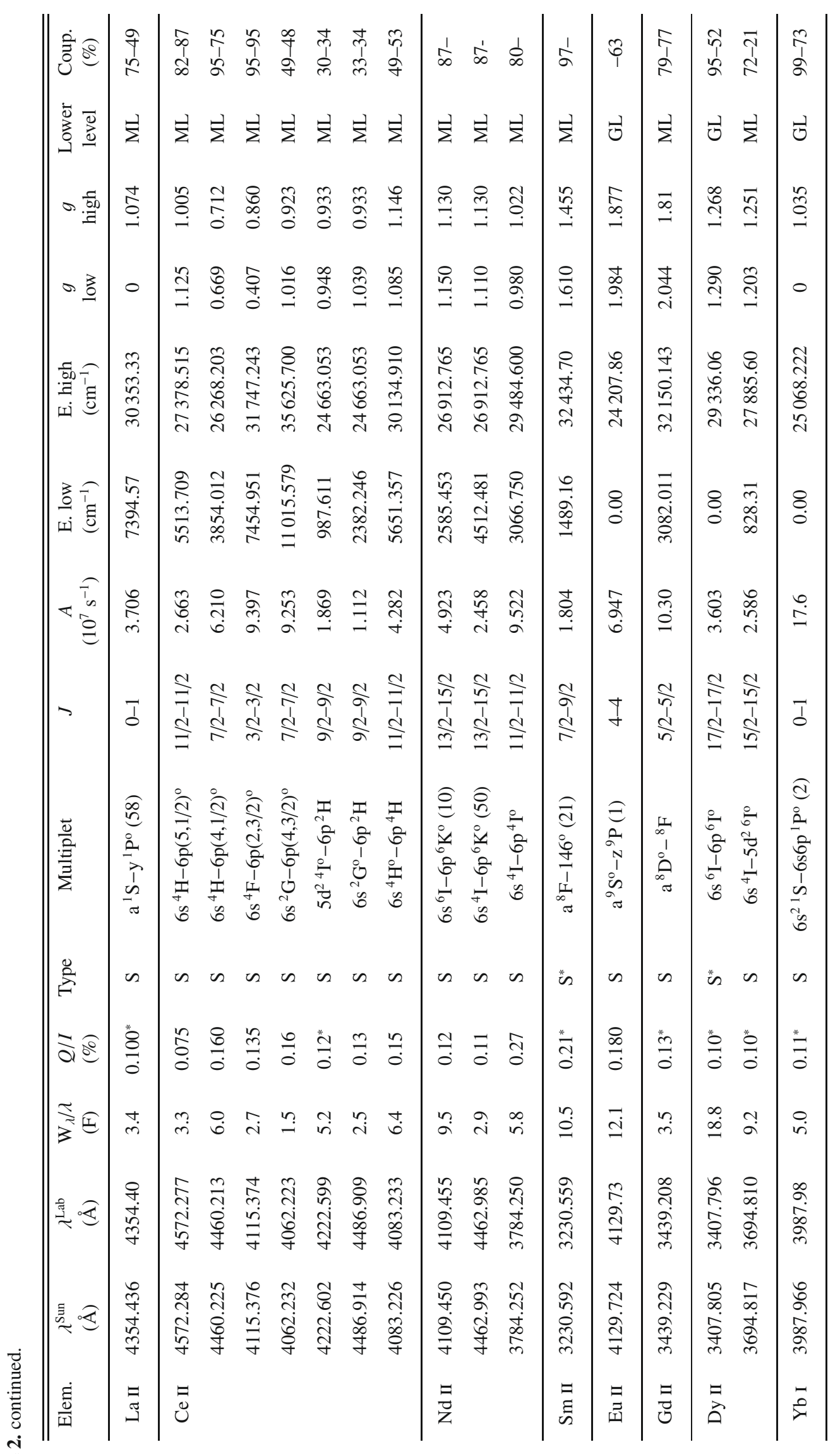

